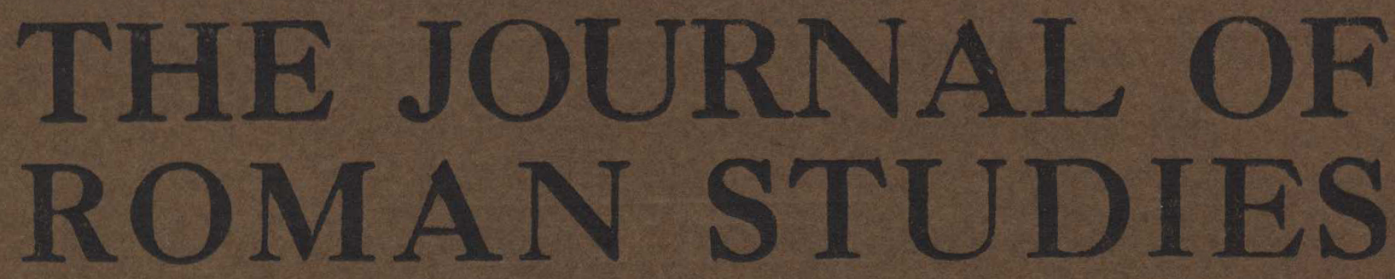

\title{
CONTENTS
}

Martin P. Nilsson. The Introduction of Hoplite Tactics at Rome : its date and its consequences. E. I. Salmon. The Pax Caudina.

Harold Mattingly. The First Age of Roman Coinage.

C. S. Walton. Oriental Senators in the Service of Rome.

Jocelyn Toynbee. The Villa Item and a Bride's Ordeal.

\section{REVIEWS OF RECENT PUBLICATIONS.}

La conquête romaine. Par A. Piganiol.

Das Haus des Herodes zwischen Jerusalem und Rom. Von Hugo Willrich.

The Roman Legions. By H. M. D. Parker.

Praetor und Formel. Von Leopold Wenger.

The culture of Ancient Greece and Rome. By F. Poland, E. Reisinger and R. Wagner. Translated by J. H. Freese,

Classical Sculpture. By A. W. Lawrence.

The classical monuments of Rome and its vicinity. Vol. I. The 'Zona archeologica.' By Giuseppe Lugli.

Numantia : die Ergebnisse der Ausgrabungen, rgo5-12. Bde. III, IV. Von Adolf Schuiten.
Dacia : an outline of the early civilisation of the Carpatho-Danubian countries. By Vasile Pârvan.

Die Trierer Kaiserthermen. Abt. I. Von D. Krencker, E. Kruger, H. Lehmann and H. Wachtler

La Verrerie Musée des antiquités nationales : Château de St. Germain-en-Laye). Par $\mathbf{R}$ Lantier.

De litteris latinis commentarii libri $\mathrm{v}$ ad criticam artis rationem exacti. Di E. De Rosa.

L. Annaei Senecae dialogorum Liber VI ad Marciam de consolatione. Par Charles Favez.

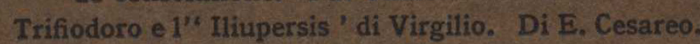

S. Ambrosii de helia et ieiunio : a commentary, with an introduction and translation. By Sister Mary J. A. Buck.

\section{NOTICES.}

A. study of the causes of Rome's wars from 343 to 265 B.C. By John William Spaeth, Jr.

La titulature impériale d'Hadrien. Par L. Perret. Rome et la Judée. Par Michel S. Ginsburg.

A manual of Eastern Roman Law-The Procheiros Nomos. Rendered into English by E. H. Freshfield.
Beschrijving van der Verzameling van het Museum G.M. Kam : de romeinsche Lampen. Door Dr. M. A. Evelein.

A bibliography of the Works of J. B. Bury, compiled, with a memoir by Norman H. Baynes.

Panorama ton Numantia. Von Harald Hofmann. Honour classics in the University of Toronto. By a group of classical graduates, with a foreword by Sir Robert Falconer.

PUBLISHED BY THE SOCIETY FOR THE PROMOTION OF ROMAN STUDIES AT THE OFFICE OF THE SOCIETY 50 BEDFORD SQUARE W.G.1. TO BE OBTAINED THROUGH ALL BOOKSELLERS AND FROM THE SOCIETY. 


\title{
The Gociety for the Promotion of ibellenic Stubies Annual Subseription - ONE GUINEA. Entrance Fee - ONE GUINEA.
}

\author{
PRESIDINT-PROFESSOR E. A. GARDNER, Litt.D.
}

OBJECTS. - The Society for the Promotion of Hellenic Studies was founded in 1879 for the following objects:-

I. - To advance the study of Greek language, literature, and art, and to illustrate the history of the Greek race in the ancient, Byzantine, and Neo-Hellenic periods, by the publication of memoirs and unedited documents or monuments in a Journal to be issued periodically.

II. - To collect drawings, facsimiles, transcripts, plans, and photographs of Greek inscriptions, MSS. works of art, ancient sites and remains, and with this view to invite travellers to communicate to the Society notes or sketches of archaeological and topographical interest.

III. - To organise means by which members of the Society may have increased facilities for visiting ancient sites and pursuing archaeological researches in countries which, at any time, have been the sites of Hellenic civilisation.

Application for membership, or for information about the Society, should be addressed to the Secretary, at 50 Bedford Square, London, W.C.I.

The Tournal of Hellenic Studies and other publications of the Society are issued by Messrs. Macmillan \& Co. Ltd, and can be purchased from any bookseller, the published price to non-members being $\ell_{2}^{2}$ 2s. net per volume or $\ell^{1}$ Is. net each part. Members are entitled to receive a copy of the Journal on publication during their membership, and can purchase back volumes at the subscription rate of $2 \mathrm{rs}$. per annum.

Members also have the use of a well-equipped library at 50 Bedford Square, and of a large collection of lantern-slides illustrative of Greek and Roman Art and Antiquities, including sets of slides. complete with texts by the best authorities on the subjects selected.

\section{The Classical Elssociation.}

The objects of the Classical Association are to promote the development and maintain the well-being of classical studies, and in particular (a) to impress upon public opinion the claim of such studies to an eminent place in the national scheme of education; $(b)$ to improve the practice of classical teaching; (c) to encourage investigation and call attention to new discoveries; (d) to create opportunities for intercourse among lovers of classical learning.

Membership of the Association is open to men and women alike. The annual subscription is $5 \mathrm{~s}$. (life composition, $f 4$ ), and there is an entrance fee of $5 s$. (not charged to Libraries). Members receive a copy of the annual Proceedings of the Association and, on payment of $2 / 6$, of The Year's Work in Classical Studies (both post free). They may also obtain the Classical Review and Classical Quarterly at reduced prices, provided that the subscriptions be paid before January 3 ist in each year. Subscriptions sent in later than that date must be at the rates offered to the general public

Inquiries and applications for membership should be addressed to the Hon. Treasurer, H. F. Hose, M.A., Dulwich College, S.E.2I ; or to either of the Hon. Secretaries, E. Norman Gardiner, D.Iitt., I, Linton Road, Oxford, and Miss E. C. Gedge, M.A., Crayford Rectory, Kent; or to the Hon. Secretary of any of the Local Branches-viz., E. D. T. Jenkins, M.A., University College, Aberystwyth: Miss M. E. Lees, M.A., University College, Bangor: C. W. Baty, M.A., 49, Waterloo Road, Bedford; G. A. Auden, M.D., M.A., F.R.C.P., F.S.A., 42. Lordswood Road, Harborne, Birmingham; W. L. Cuttle, M.A., The University. Bristol : J. E. Scott, M.A., Caius College, Cambridge ; L. J. D. Richardson, M.A., Fernshaw. Hilary Road, Llanishen, Cardiff; M. M. Gillies, Ph.D., The University, Hull; Miss Towne, County School for Girls, Beckenham, Kent; D. E. F. Binyon, The University, Leeds; Miss A. I. S. Smith, Girls' Grammar School, Bradford; R. B. Onians, Ph.D., 35, Mount Street, Liverpool: Miss E. J. Vaughan, B.A., University College, W.C.I : W. Beare, M.A., The University, Manchester; B. Anderton, M.A., Public Library, Newcastle-on-Tyne; Professor F. S. Granger, University College, Nottingham; T. B. L. Webster, M.A., Ch. Ch. Oxford; Miss N. C. Jolliffe, The University, Reading; Mrs. Jackson, The Cottage, Cordwell Valley, Holmesfield, Nr. Sheffeld; Miss M. K. Norris, B.A., University College, Southampton; F. Fletcher, M.A., University College, Exeter: Miss W. A. Odell, Brighton and Hove High School for Girls; H. Hill, M.A., University Coll., Swansea. 


\section{THE JOURNAL OF ROMAN STUDIES}


All rights reserved. 


\section{THE JOURNAL OF ROMAN STUDIES}

\section{VOLUME XIX}

PUBLISHED BY THE SOCIETY FOR THE PROMOTION OF ROMAN STUDIES AT THE OFFICE OF THE SOCIETY, 5O BEDFORD SQUARE, W.C.1. LONDON

1929 
The printing of this part was completed on Fuly 16,1930 


\section{CONTENTS.}

Martin P. Nilsson. The Introduction of Hoplite Tactics at Rome: its date and

PAGT:

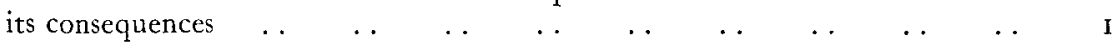

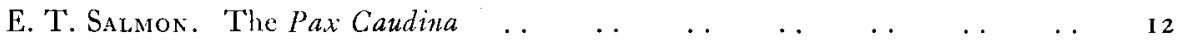

Harold Mattingly. The Fïrst Age of Roman Coinage $\ldots \begin{array}{llllll} & \ldots & \ldots & \ldots & \text { I9 }\end{array}$

C. S. Walton. Oriental Senators in the Service of Rome . $\quad \begin{array}{llllllllll} & \ldots & \ldots & \ldots & 38\end{array}$

Jocelyn Toyn bee. The Villa Item and a Bride's Ordeal . .

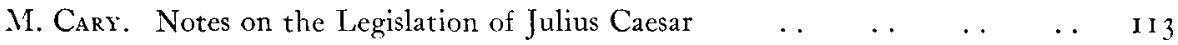

Felix Oswatd. Bowls by Acavnissa from Birdoswald, Mlainz and Cologne . 120

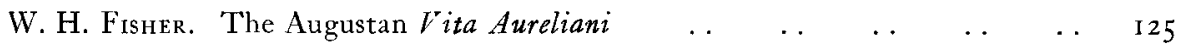

T. B. I. Webster. The Wilshere Collection at Pusey House in Oxford .. $\quad \ldots \quad 150$

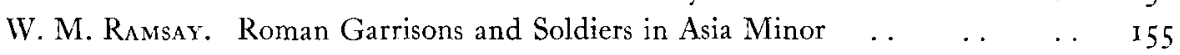

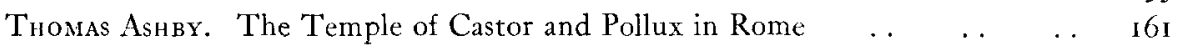

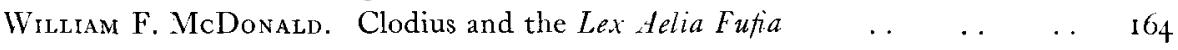

M. V. TAYlor and R. G. Collingwood. Roman Britain in $1929 \quad \ldots \quad \ldots \quad$ I80

$\begin{array}{llllllllll}\text { Reviews and Discussions } & \ldots & \ldots & \ldots & \ldots & \ldots & \ldots & \ldots & \ldots & 219\end{array}$

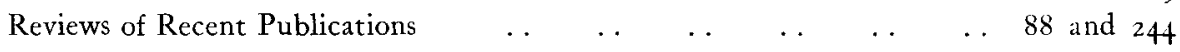

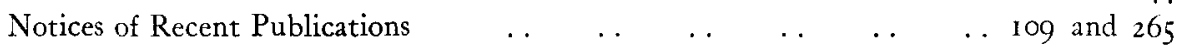

List of Works received ..

Proceedings of the Society for the Promotion of Roman Studies, 1929-30: open $\begin{array}{llllllllllll}\text { meetings } & . & \ldots & \ldots & \ldots & \ldots & \ldots & \ldots & \ldots & \ldots & \ldots & 270\end{array}$

$\begin{array}{llllllllll}\text { Annual General Meeting } & \ldots & \ldots & \ldots & \ldots & \ldots & \ldots & \ldots & \ldots & 270\end{array}$

Report of the Council for the year ending 3Ist December, $1929 \quad \ldots \quad \ldots \quad \ldots \quad 271$

Income and Expenditure Account for the year ending 31st December, 1929 $\quad \ldots \quad 274$

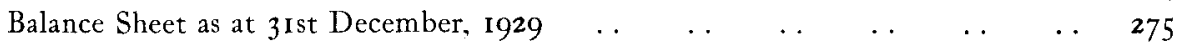

$\begin{array}{lllllllllllll}\text { Index } & \ldots & \ldots & \ldots & \ldots & \ldots & \ldots & \ldots & \ldots & \ldots & \ldots & \ldots & 277\end{array}$

$\begin{array}{llllllllllll}\text { Title Page } & \ldots & \ldots & \ldots & \ldots & \ldots & \ldots & \ldots & \ldots & \ldots & \ldots & \text { ii }\end{array}$

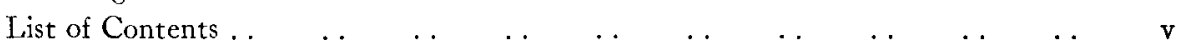

$\begin{array}{llllllllll}\text { List } \text { of Reviews and Discussions } & \ldots & \ldots & \ldots & \ldots & \ldots & \ldots & \ldots & \text { vi }\end{array}$

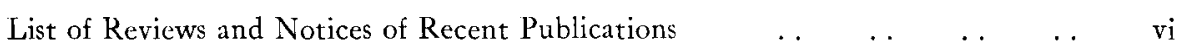

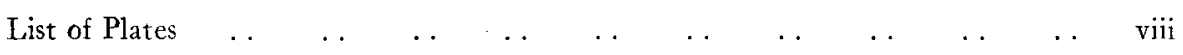

List of Maps and of Illustrations in the Text $\quad \ldots \quad$.

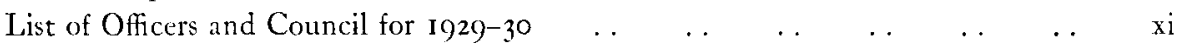

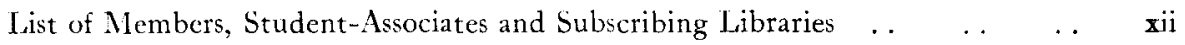




\section{REVIEWS AND DISCUSSIONS.}

Die Augustus-inschrift auf dem Markplatz von Kyrene. Von Johannes Stroux u.

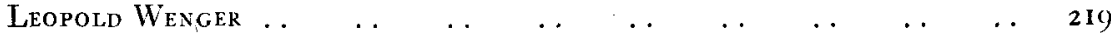

La Fin du Monde Antique et le Début du Moyen Àge. Par Ferdinand I،ot .. 224

Les Villes du Moyen Age, Essai d'histoire économique et sociale . . $\quad \ldots \quad \ldots \quad \ldots \quad 224$

The Social and Economic History of the Roman Empire. By M. Rostovtzeff . . 224

Publii Ovidii Nasonis Fastorum Libri Sex. Ed. with a translation and commentary

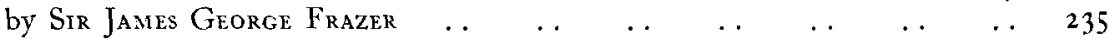

Sexti Properti quae supersunt opera. Ed. Oliffe L. Richmond $\quad \ldots \quad \ldots \quad \ldots \quad \ldots$

\section{REVIEWS AND NOTICES OF RECENT PUBLICA'TIONS.}

REVIEWS.

La conquête romaine. $\begin{array}{llllllllll}\operatorname{Par} A . \text { Piganiol } & \ldots & \ldots & \ldots & \ldots & \ldots & \ldots & 88\end{array}$

Das Haus des Herodes zwischen Jerusalem und Rom. Von Hugo Willrich _. 90

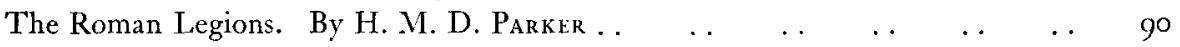

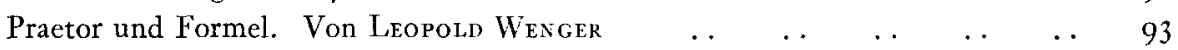

The Culture of Ancient Greece and Rome. By F. Polaxd, F. Reisinger and

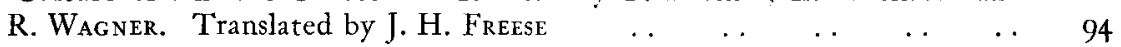

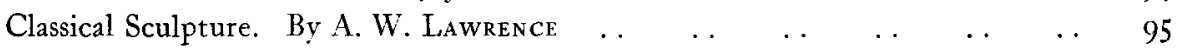

The Classical Monuments of Rome and its vicinity. By Gruseppe Lugli $\quad \ldots \quad 98$

Numantia : die Ergebnisse der Ausgrabungen, 1905-12. Bande iii, iv . . . . 99

Dacia: An outline of the early civilisations of the Carpatho-Danubian Countries.

By Vasilue Pàrvan ..

Die Trierer Kaiserthermen. Abt. I. Von D. Krenkcer, E. Krüger, H. Lenuan N and H. WAchtLER ..

La Verrerie (Musée des Antiquités nationales: Château de Saint-Germain-en-

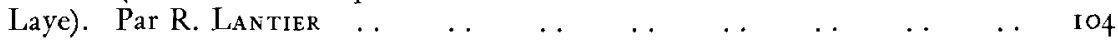

De Litteris Latinis Commentarii Libri $V$ ad criticam artis rationem exacti. Di

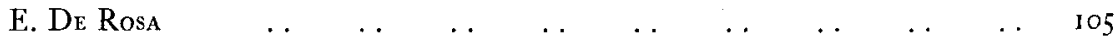

L. Annaei Senecae Dialogorum Liber VI ad Marciam de Consolationc. Par $\begin{array}{llllllllllll}\text { Charles Favez }_{\text {F }} & \ldots & \ldots & \ldots & \ldots & \ldots & \ldots & \ldots & \ldots & \ldots & 106\end{array}$

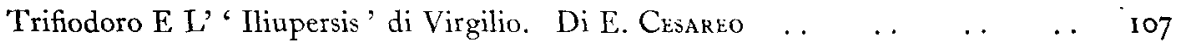

S. Ambrosii de Helia et Ieiunio: A commentary, with an Introduction and

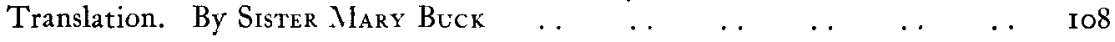

The Cambridge Ancient History, vol. vii $\quad \ldots \quad \begin{array}{llllllll} & \ldots & \ldots & \ldots & \ldots & \ldots & 244\end{array}$

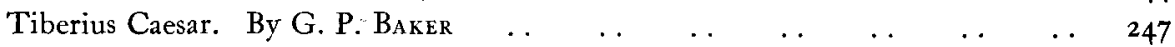
Eine Gerichtsreform des Kaisers Claudius (B.G.U. 6I I). Von Jonannes Stroux 248 
PAGE

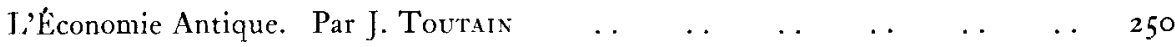
The Economic Life of the Ancient World. By Jules Toutain $\ldots \quad \ldots \quad \ldots \quad 250$ Pagan Regeneration. By Harom R. Willoughby . . . . $\quad \ldots \quad \ldots \quad \ldots \quad \ldots \quad 25$. A Handbook of Greek and Roman Architecture. By D. S. Robretsox . . . $\quad 252$

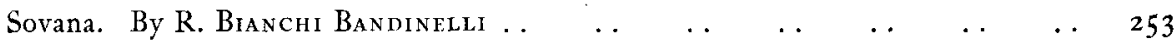

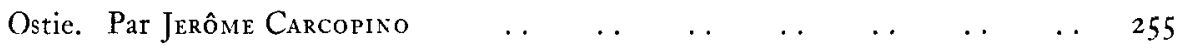

I.e Vie Maestre d'Italia: Via Flamina, studio storicotopografico. Di Edoardo

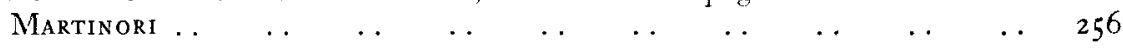

Dacia, Recherches et découvertes archéologiques en Roumanie I and II. Ed. by

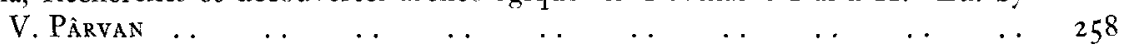

Second Report upon the Fxcavations carried out in and near the Hippodrome of Constantinople in I928 on behalf of the British Academy . . . . . 260

Ein Römischer Bauernhof bei Mayen. Von Iiranz Oelmaxx $\quad \ldots \quad \ldots \quad \ldots \quad \ldots \quad 26 \mathbf{I}$

Geschichte der Romischen Titeratur. Von Martix Schanz $\quad \ldots \quad$. $\quad \ldots \quad \ldots \quad 263$

Virgile et le mystère de la IVe. Fglogue. Par Jerôme Carcopino $\quad \ldots \quad \ldots \quad \ldots \quad 264$

\section{NOTICES.}

A Study of the Causes of Rome's Wars from 343 to 265 в.c. By J. IV. Spaftir, Jr. Io9

La Titulature Impériale d'Hadrien. Par L. Perret $\quad \ldots \quad \ldots \ldots \ldots$

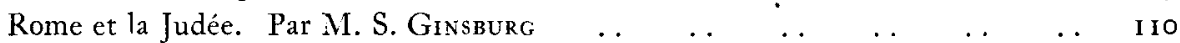

A Manual of Eastern Roman Law-The Procheiros Nomos. Rendered into

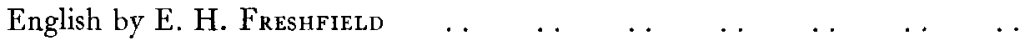

Beschrijving van der verzameling van het Museum G. M. Kam : de Romeinsche

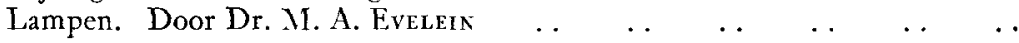

A Bibliography of the works of J. B. Bury, compiled, with a memoir. by Norman $H$.

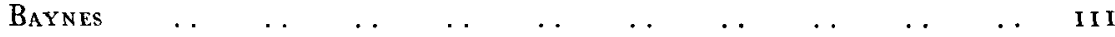

Panorama von Numantia. Von Harald Hofman $\quad \ldots . \quad \ldots \quad \ldots \quad \ldots \quad$ II I

Honour Classics in the University of Toronto. By a group of classical graduates, with a foreword by Sir Robert Falconer $\ldots \quad \ldots \quad \ldots \quad \ldots \quad \ldots \quad \ldots \quad$ II I

A Survey of Ancient History. By M. L. W. Latstiver $\quad \ldots \quad \ldots \quad \ldots \quad \ldots \quad \ldots .265$

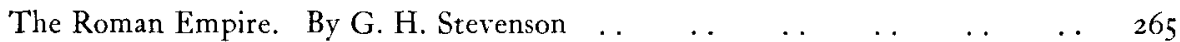
Psychology, ancient and modern. By G. S. Brett $\ldots \begin{array}{llllllll} & \ldots & \ldots & \ldots & \ldots & & 266\end{array}$

Heerwesen und Kriegfuhrung der Griechen und Römer. Von Jonaxyes Krowayer und GeORG VeITH

Inscriptions Grecques et Latines de la Syric. Par Locis Jalabert et Rexé

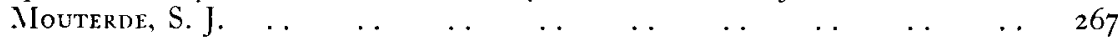

The Elder Pliny's Chapters on Chemical Subjects. Pt. I. Ed. Kenseth C. Balley 267

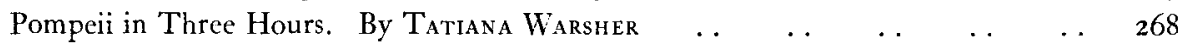

The Prehistoric and Roman Remains of Denbighshire. By the Rer. Elus Davies 268 


\section{LIST OF PLATES.}

The First Age of Roman Coinage:

Plate I. Romano-Campanian and kindred coins: Capua.

Plate II. Atella, Calatia : Roman Brettii: Carthaginian: Victoriates.

Plate ir. Aes signatum.

Bowls by Acavnissa from Birdoswald, Mainz and Cologne :

Plate rv. Facsimile of decoration and signatures.

The Wilshere Collection at Pusey House in Oxford :

Plate v. Gold Glass at Pusey House, Oxford.

I. In the centre, busts of husband and wife ; round the outside, biblical scenes.

2. Man and woman praying.

Plate vi. I. In the centre, SS. Peter and Paul; round the outside, biblical scenes.

2. Christ and eight Saints.

Roman Britain in 1929 :

Plate vir. Caerleon I Section across the NW. defences.

2. Section across the SW. defences.

Plate vir. Types of tile antefixes found during the cxcavation of the Prysg Ficld. Caerleon.

Plate Ix. Silver plaque found in Moorgate Street, London.

Plate x. Camp in Lydney Park, Glos.

Plate xr. Bronze figure of an Irish wolf-hound found at Lydney.

Plate xir. Fibulae, tweezers, and seal-boxes found at Somerdale.

Plate xirr. Iron and bronze fibulae found at Kingsdown Camp. Mells, Somerset

Plate xiv. Bronze fibulae found at Kingsdown Camp, Somerset.

Plate xv. Thatcham, Berks: six pewter vessels, with pottery, padlock and spindlewhorl found in a Roman well.

Plate xvi. The Roman villa, Ashtead Common, Surrey.

Plate xvir. Richborough : the inner (Claudian) ditch between the west gate of the Saxon-shore fort and the great foundation, showing the later roads passing over the filling.

Plate xvill. Richborough : abutments of bridge carrying the road over the inner of the external ditches of the Saxon-shore fort.

Plate xIx. Birdoswald: slab used as a paving-stone in the Theodosian floor of a barrack-building.

Plate xx. Caerleon, the Prysg Field: leaden dies found in 1928 and 1929. 
The First Age of Roman Coinage :

Fig. I.

Fig. 2.

The Villa Item and a Bride's Ordeal :

Fig 3. Plan of the triclinium in the Villa Item, Pompeii.

Fig. 4. Sketch of the Frescoes in the Villa Item, Pompeii.

Fig. 5. Sketch of the Frescoes in the Villa Item, Pompeii.

Fig. 6. Sketch of the Frescoes in the Villa Item, Pompeii.

Bowls by Acavnissa from Birdoswald, Mainz and Cologne :

Fig. 7 .

Roman Britain in 1928 :

Fig. 8. New Earswick, 1928.

Fig. 9 .

Fig. 10 .

Fig. 11. Pottery found below the fioors of the Roman villa on Ashtead Common.

Fig. 12. Ashtead: the actual state and a reconstruction of a chimney-pot, and an enlarged column of tiles.

Fig. 13. Richborough: general plan.

Fig. I4. No. 12. 


\section{SOCIETY FOR THE PROMOTION OF ROMAN STUDIES.}

\section{OFFICERS AND COUNCIL FOR 1930-1931.}

\section{President.}

PROF. F. E. ADCOCK, D.LITT. M.A.

\section{Vice-Presidents.}

PROF. J. G. C. ANDERSON, M.A. THOMAS ASHBY, M.A. F.B.A. D.LITT. F.S.A. N. H. BAYNES, M.A.

R. C. BOSANQUET, M.A. F.S.A.

SIR A. J. EVANS, D.LITT. F.R.S. F.S.A. F.B.A.

G. F. HILL, LL.D. LITT.D. F.B.A.

T. RICE HOLMES, D.LITT. LITT.D.

PRINCIPAL H. STUART JONES, D.LITT. F.B.A. SIR FREDERIC KENYON, G.B.E. K.C.B. D.LITT.

LITT.D. LL.D. F.B.A.
H. M. LAST, M.A.

SIR G. MACDONALD, K.C.B. D.LITT. LL.D. F.B.A. J. W. MACKAIL, LL.D. F.B.A.

L. C. PURSER, LITT.D.

SIR W. M. RAMSAY, D.LITT. D.C.L. LL.D. F.B.A. THE RIGHT HON. SIR J. RENNELL RODD, G.C.M.G. G.C.V.O. C.B.

A. H. SMITH, M.A. F.S.A.

MRS. ARTHUR STRONG, C.B.E. LITT.D. LL.D. F.S.A.

\section{Ordinary Members of the Council.}

Elected June, 1928.

SIR JOHN BARRAN, BART.

W. PARKER BREWIS, M.A. F.S.A.

C. W. M. $\operatorname{CoX}$, M.A.

E. NORMAN GARDINER, D.LITT.

ROBERT GARDNER, M.A

V. E. NASH-WILLIAMS, M.A.

T. DAVIES PRYCE, M.R.C.S. F.S.A.

G. MCN. RUSHFORTH, M.A. F.S.A.

Elected June, 1929.

PROF. W. BLAIR ANDERSON, LITT.D.

R. H. BARROW, M.A.

M. P. CHARLESWORTH, M.A.
MISS M. L. GORDON.

R. P. LONGDEN, M.A.

J. G. MILNE, D.LITT.

G. H. STEVENSON, M.A.

R. E. M. WHEELER, D.LITT. F.S.A.

PROF. B. ASHMOLE.

Elected June, 1930.

E. B. BIRLEY, M.A.

PHILIP CORDER, M.A.

ROGER HINKS.

H. C. OAKLEY, M.A.

C. A. R. RADFORD, M.A. F.S.A.

PROF, H. J. ROSE.

E. WOOLLEY, F.S.A.

Treasurer.

Auditor.

ROBERT HOLLAND-MARTIN, C.B. F.S.A.

C. T.[EDGE, M.A. A.C.A.

\section{Editorial Committee.}

PRINCIPAL H. STUART JONES. SIR G. MACDONALD.

PROF. F. E. ADCOCK.

H. M. LAST.

Keeper of Books and Lantern-slides.

JOHN PENOYRE, C.B.E. M.A. 50 Bedford Square, W.C.1.

Secretary and Editor.

MISS M. V. TAYLOR, M.A. F.S.A. Haverfield Library, Ashmolean Museum, Oxford. 


\title{
LIST OF MEMBERS.
}

To July Ist, 1930.

\begin{abstract}
N.B.-It is requested that notice be given to the Secretary of any error, omission, change of address, resignation or death.
\end{abstract}

\section{HONORARY MEMBERS.}

R. Cagnat, Professeur au Collège de France, Secrétaire perpetuel de l'Académie des Inscriptions et Belles Lettres, 3 rue Mazarine. Paris, VI $I^{e}$.

Professore Gaetano De Sanctis, Via Santa Chiara 6I, Roma, Italy.

Professor Dr. Ernst Fabricius, Goethestrasse 44, Freiburg-i-Breisgau, Germany.

Professor Dr. Wilhelm Kubitschek, Pichlergasse I, Wien, IX, Austria.

Professor M. Rostovtzeff, Yale Station 1916, Nesv Haven, Conn., U.S.A.

\section{ORDINARY MEMBERS.}

$\mathrm{L}$ indicates Life Compounder.

L Abbott, Edwin, M.A. Fesus College, Cambridge.

Adams, P. H., F.R.I.B.A. Pembridge Manor Hotel, Pembridge Crescent, $W^{11}$.

Adcock, Prof. F. E., D.Litt., M.A. King's College, Cambridge.

Ainslie, Miss G. Sedber, Sberborne, Dorst t.

Aitchison, W. de L. Killingworth Hall, Northumberland.

Alford, Miss. 5I Gloucester Gardens, Bishop's Road, $W .2$

L Allen, P. S., M.A., D.Litt., F.B.A. Corpus Cbristi College, Oxford.

Alles, Dr. F. R. British Hotel, Colombo, Ceylon.

Alton, Prof. E. H, M.A. 4o Trinity College, Dublin.

Anderson, Prof. J. G. C, LL.D. 25 Charlbury Road, Oxford.

Anderson, R. H. 95 Alexandra Road, N.W. ${ }^{\mathrm{s}}$

Anderson, Prof. W. Blair, Litt.D. The University, Manchester.

Anderson, Prof. W. C. F, M.A. Hermits Hill, Burghfield Common, Reading.

Andrews, P. M., A.R.I.B.A. $63 \mathrm{~W}$ ellesley Avenue, Iver, Bucks.

Armstrong, G. C. Levair, St. Fohn's Road, Cosham, Hants.

L Ashby, Thomas, M.A., D.Litt., F.B.A., F.S.A. ${ }_{4}$ Via Vincenzo Bellini, Rome (36), Italy.

Ashley, Miss A. M. 7 Burbage Road, S.E. ${ }^{24}$

Ashmole, Prof. B., M.A. Cbesterfield, Meads, Eastbourne.

Atkinson, Prof. D., B.A. The University, Manchester.

Austin, E. H. $\quad$ I58 Poplar Road, Merton Park, S.W. ${ }^{19}$

Baddeley, St. Clair. Castle Hale, Painswick, Stroud, Glos. Bagenal, Hope, A.R.I.B.A. 34 Bedford Square, W.C.1

Bagnani, Gilbert. 4 Via S. Martino al Macao, Rome, Italy.

Bailey, C, M.A. Balliol College, Oxford.

Bailey, W. O. Faircroft, Cobbam, Surrey.

Baillie Reynolds, P. K.,.M.A., F.S.A. The University College of $W$ ales, Aberystovth.

Baker, A. B. Lloyd, B.A. Devonsbire House, Batb Road. Cheltcnham.

Baker, G. P. Marigold Corner, Elmer, Bognor Regis, Sussex.

Baker, T. T. 44 Burnbam Court, Bayswater, $W .^{2}$

Baldwin, The Rt. Hon. Stanley, M.A., M.P. Astlcy Hall, near Stourport. 
Balsdon, J. P. V. D. Exeter College, Oxfurl.

Barber, E. A. M.A. Exeter College, Oxford.

L Barlow, Miss A. E. F. Greenthorne, Edgworth, near Bolton.

Barnett, T. G. Rednal, Birmingham.

Barran, Sir John, Bart. Suzoley Hall, Ripon.

Barrow, R. H. 24 Hilldown Road, S.W. ${ }^{16}$

Barton, Mrs. W. J., M.A. I9 Penny Street, Portsmouth

Basden, Miss M. F. 2I The Drive, Hove.

Batchelor, F., M.A. The Glasgow Academy, Glasgow, $W .{ }^{2}$

Baty, C. W., M.A. Greenbank House, 5 Cambrian View, Chester.

Baynes, N. H, M.A. Fizzwaiters, Nortbwood, Middlesex.

L Benecke, P. V. M, M.A. Magdalen College, Oxford.

L Benn, Mrs. Alfred. Il Ciliegio, Via del Palmerinn, Fiorence, Itaiy.

Bennett. S. A. Warreleigh, Budleigh Salterton, Devon.

Bensiy, Prof. E, M.A. Oundle Cottage, Much Hadbam, Herts

Bentinck, 1. Cavendish, F.S.A. 78 Harley Street, $W .{ }^{1}$

Benton, W. A. 30 Wretham Road, Handsworth, Birmingham.

L Berry, Sir James, Kt., F.R.C.S. F.S.A. Bramblebury, Dunsmore, nr. Wendover, Bucks.

Bettoney, H. $7 \mathrm{r}$ Romney Street, Westminster, S.W. ${ }^{1}$

Bevan, E. R. Wray Lane House, Reigate.

L Billson, C. J., M.A. Weald House, Heathfield, E. Sussex.

Birley, E. B., M.A. Moorland, Didsbury, Manchester.

Blackman, Miss J. E. S. St. Foan, Barnett Wood Lane, Asbtead, Surrey.

Bland, Miss E. D. 26 Luttrell Avienue, Putney, S. $W^{15}$

Blegen, Mrs. C. W. 9 Plutarch Street, Athens, Greece.

Blomfield, Sir Reginald, M.A. R.A. F.S.A. 51 Frognal, Hampst:au, N.W."

L Body, L. A., M.A. St. Chad's College, Durbam

L Bosanquet, R. C, M.A., F.S.A. Rock Mour, Alnwick.

Bothamley, C. H., M.Sc. Resthaven, Yatton, Somerset.

Bowen, H. C. Caldy View, Tenby, S. Wales.

Bowra, C. M., M.A. Wadham College, Oxford.

Box, H., B.A. 485 King Street, Aberdeen.

Boyce, Sidney M., B.A. The Grammar Scbool, Chorley.

Braunholtz, Prof. G. E. K. 22 Old Road, Headington, Oxon.

Brett, Mrs. A. B, M.A., F.R.N.S. 13 I Maple Avenue, Flusbing, N.Y., U S.A.

Brewis, W. Parker, F.S.A. Glenbrae, Fesmond Park West, Newcastle-on- $\mathcal{T}_{y}$;ne.

Brice-Smith, R, M.A. Brigbtlands, Newnbam, Gloucestersbire.

Bridge, J. J. R. I Bloomfield Place, Bath.

Brigg, W. Anderton, M. A. LL.M. Kildwick Hall, nr. Keigbley, Yorks.

Brighouse, T. K. Lower Wild Carr, Pateley Bridge, Harrogate.

Brooke, Rev. A. E, D.D. King's College, Cambridge.

Brown, A. Theodore. 25 Lord Street, Liverpool.

Brown, Prof. G. Baldwin, M.A. The University, Edinburgh.

L Brown, James. Gala Hill, Galashiels.

Brown, W. B. Io Corfton Road, Ealing, $W$.

Bruce-Clarke, Miss. Minley, Reigate.

Brydges, E. E. H, M.A. II Stone Buildings, Lincoln's Inn, W.C.:

Buckland, Prof. W. W, Litt.D. 9 Grange Terrace, Cambridge.

Buckler, W. H., M.A. I Bardwell Road, Oxford.

L Bulwer, Miss. Dalling, Nr. Uckfield, Sussex.

L Bulwer, Miss Dora E. Dalling, Nr. Uckfield, Sussex.

L Burdon, Rev. R. J. Parkburst House, Haslemere.

Burnside, Rev. Canon W. F, M.A. St. Edmund's School, Canterbury.

Burrowes, Major P. W. Mont au Nord, ferbourg, Guernsey.

Bury, Gerard. West Wratting Park, Cambridge.

L Bush, W. T. Columbia University, New York City, N.r., U.S.A. 
Bushe-Fox, J. P., F.S.A., Benlomond House, Dounshire Hill, Hampstead, N.W.

Butler, Prof. H. E, M.A. University College, London.

Butler, Harold. Heston Cottage, Heston, Middlesex.

L Buxton, Denis A. J. 43 Campden Hill Square, $W .{ }^{8}$

Calder, Prof. W. M, M.A. The University, Mancheszer.

Callaghan, Rev. T. T. c/o Messrs. Bickers छ Sons, Charles Street, Haymarket, W.

Callender-Brown, W. H., M.A. Haledene, Edowarebury Lane, Edgware, Middlesex.

Cameron, A., M.A. The Humanity Dept., The University, Edinburgh.

Cameron, Prof. D. J, M.A. Anderson House, Anderson Road, Colombs.

Capps, Prof. E. Princeton, New Fersey, U.S.A.

Carlisle, Mrs. M. Haverbrack House, Milntborpe, Westmorland.

L Caroë, W. D, M.A. F.S.A. 3 Great College Street, Westminster, S.W.'

$\mathbf{L}$ Carthew, Miss. 6 Albert Place, Victoria Road, $W .{ }^{8}$

Ca y, M, D.Jitt. 82 Talbot Road, Highoate, N.6

Caspersz, A., B.A. Polemead, Cranleigh, Guildford.

Chance, Arthur F. The Schools, Shrewsbury.

Chapman, C. S., M.A. The Bede School for Boys, Sunderland.

Charlesworth, M. P., M.A. St. Jobn's Collegs, Cambridge.

Chitty, Rev. G. J, M.A. Eton College, Windsor.

Clark, Prof. A. C, M.A. Litt.D. F.B.A. Corpus Christi College, Oxford.

Clark, J. 3 Spring Gardens, Glasgow, N.W.

Kitsun-Clarke, Col. Leeds.

L Clauson, Sir Charles. Hawkshead House, Hatfield.

Cleminson, F. J. 32 Harley Street, $W .1$

Clifton, F. W. V, M.A. II Mealuz Bank, Chorltoncille, Mantester.

Cloke, F. A. Salisbury House, 5 I Strand Street, Sandwich.

Coate, Miss Dora. The High Scbool, Truro.

Cole, S. C, B.A. 32 Hampton Park, Redland, Bristol

Colegate, A. I6 Princes Gardens, S.W.'

Coleman, R. H. F. New College, Harrogate.

Coleman, W. H., B.A. 22 Elgin Park, Redlands, Bristol.

Collingwood, R. G, M.A. F.S.A. Pembroke College, Oxford.

Collison, C. Lonsdale House, St. Bees, Cumberland.

Connall, Prof. B. M, M.A. 7 Claremont Drive, Headingley, Leeds.

Conway, Prof. R. S, M.A., Litt.D., F.B.A. Draethen, Didsbury, Manchester.

Cook, A. B, M.A. Queens' College, Cambridge.

Cooke, Rev. J. C, B.A. St. Bede's College. Mlanchester.

Cookson, C, M.A. Magdalen College, Oxford.

Corder, Philip, M.A. 4 Bootham Terrace, Tork.

Corley, F. E. Highfield, Witney, Oxon.

Cornford, Henley. 59 Ranelagh Gardens Mansions, S.W. ${ }^{6}$

Cornford, F. M, M.A. Conduit Head, Madingley Road, Cambridge.

Cotton, A. R., M.B.E., F.S.A. The White House, Epsom.

Cox, C. W. M., M.A. New College, Oxford.

Craig, J. D, M.A. The University, Sheffield.

Craster, H. H. E, M.A., D.Litt., F.S.A. All Souls College, Oxford.

Crees, Dr. J. H. E. The Cathedrai School, Herford.

Cripps, Mrs. Wilfred. Cripps Mead, Cirencester.

Crocker, A. J. 38 Cromwell Road, Felthar, Middlesex.

Crossley, Miss V. G., M.A. Downs Scbool, Seaford.

Culley, Mrs. Willougbby, Wildrwood Roal, Golders Green, N.W.'1

L Cumont, Prof. F. I9 Corso d'Italia, Rome, Italy. 
Cunnington, Mrs. M. E. 33 Long Street, Devizes.

Curle, James, LL.D., F.S.A. Priorwood, Melrose.

Dakyns, G. D, M.A. The White House, Iffley, Oxford.

Dalton, O. M, M.A., F.B.A, F.S.A. I2 Sydney Place, Bath.

Daniel, A. M. 2 Hampstead Hill Gardens, Rosslyn Hill, N.W. ${ }^{3}$

Danson, J. R., M.A., M.C. 74 Bidston Road, Birkenhead.

Dare, M. Paul. 6 Lincoln Street, Loicester.

Davis, S. C. H. 20 Blenbeim Road, St. 'Fobn's Wood, N.W. ${ }^{8}$

Davison, J. A. Classics Dept., The University, Manchester.

Dean, Prof. L. R. Denison University, Obio, U.S.A.

Dennis, C. P. L., M.A., Ph.D.

L Deubner, Prof. L. 55 Friedrich Wilhelmstrasse, Scblachense, Berlin, Germany.

L de Zulueta, Prof. F, M.A. D.C.L. New College, Oxford.

Dickson, Mrs. Gæorge. Sesame Club, Grosvenor Street, $W .1$

Dodd, E. E, M.A. 20 Sheriff Lane, Eldwick, Bingley, Yorks.

Dodd, P. W, M.A. Fesus College, Oxford.

Drake, H. L, M.A. Pembroke College, Oxford.

Droop, Prof. J. P. 23 Ullet Road, Sefton Park, Liverpool.

Drummond, Rev. W. H. I5 Rawlinson Road, Oxford.

Duff, J. D., M.A. Strathaird, Cambridge.

Duff, Prof. J. Wight, M.A., Litt.D. Armstrong College, Newcastle-upon-Tyne.

Dunning, Major J., D.S.O. $3 \mathbf{I}$ Lombard Street, E.C. ${ }^{4}$

Duxfield, Miss M. I 3 Asb Street, Soutbport, Lancs.

Earl, G. C. 2914 McClure Street, Oakland, California, U.S.A

Eastman, Mrs. Charlotte. Lowa City, Iowa, U.S.A.

Ecob, P. E., M.A. Bedford Road Collegiate Institute, Saskatoon, Saskatchewan, Canada.

Edge, Cyril T, M.A., A.C.A. Vernon House, Sicilian Avenue, Soutbampton Row, W.C. ${ }^{1}$

Edmonds, J. M, M.A. Gryt-Howe Cottage, Storey's Way, Cambridge.

Egerton-Warburton, W., M.A. C/o The War Office, London, S.W. ${ }^{1}$

Elliot, Capt. W., R.F.A. 28 Thornbill Gardens, Sunderland.

Estelrich, J. Apart, 789, Barcelona, Spain.

L Evans, Sir A. J, M.A., D.Litt., LL.D., F.R.S., F.B.A., F.S.A. Youlbury, near Oxford.

Evans, Sir E. Vincent, K.C., C.H., LL.D., F.S.A., $6_{4}$ Chancery Lane, W.C. ${ }^{2}$

Evans, Lady, M.A. 9 Kensington Park Gardens, $W .11$

Evans, Prof. D. Emrys. University College, Singleton Park, Swansea.

Ewing, James L, LL.D. Derreen, Murrayfield, Edinburgt.

Fair, Miss M. C. 2 Rigg Cottages, Eskdale, Cumberlanu.

Farquhar, James. Secondary School, Leamington-on-Tyne.

Fell, Rev. R. A. L. Kolbar, Abmednager District, India.

Fisher, Rev. G. F, M.A. The Hall, Repton, Derby.

L FitzHugh, Prof. Thomas. University of Virginia, Cibarloatesville, Fa. U.S.A.

L Fleming, Miss Halen J. Caerlee, Innerleithen, Peebleshire.

Flemming, Prof. Percy, F.R.C.S. 89 North Gate, N.W. ${ }^{8}$

Fletcher, Sir Banister, P.R.I.B.A., F.S.A. I King's Bench Walk, E.C. ${ }^{4}$

Fletcher, Frank, M.A. 48 Polsloe Road. Exeter.

Fletcher, G. B. A. The University, Liverpool.

Flood, Rev. P. J., D.D. The Presbytery, Dabbeth, Tollerose, Glasgow. 
Fordyce, C. J., M.A. Tesus College, Oxford.

Forsey, G. F. 4 Glebe. Court, Highfield Lane, Soutbampton.

Fowler, Rev. J. R. Curepipe, Mauritius.

Fox, Cyril, Ph.D., F.S.A. Four Elms, Heol Wen, Rbiwbina, Cardiff.

Francis, A. G, O.B.E., B.A., M.B., B.Sc., F.R.C.S. 20 Beacon Hill, N. ${ }^{7}$

Frank. Professor Tenney. Jobns Hopkins University, Baltimore, Maryland, U.S.A.

Franks, Percy, M.A. De Aston Grammar Scbool, Market Rasen.

Frazer, Sir J. G., O.M., LL.D., D.Litt., Litt.D., D.C.L., F.B.A. Oueen Anne's Mansions, St. Fames Park, S.W. ${ }^{1}$

Fremantle, Miss I. M. E. Huguenot University College, Wellington, Cape Province, S. Africa.

Freshfield, Douglas W, M.A. D.C.L. F.R.G.S. Wych Cross Place, Forest Row, Sussex.

Freshfield, E. H. Mint House, Upper Galton, Reigate.

Gair, Mrs. H. B. I 10 Goldburst Terrace, N.W.6

Gardiner, E. Norman, D.Litt. I Linton Road, Oxford.

Gardner, Prof. E. A., M.A. Recess, Boyn Hill, Maidenbead.

Gardner, Prof. ظ. G, M.A. 5 Ruskin Clnse, Meadway, $N W .11$

Gardner, Prof. Percy, LL.D., Litt.D., F.B.A., F.S.A. I2 Canterbury Road, Oxford.

Gardner, Eric, M.B., F.S.A. Portmore House, Weybridge.

Gardner, R, M.C. M.A. Emmanuel College, Cambridge.

Gardner, Willoughby, D.Sc., F.S.A., F.L.S. Deganwy, N. Wales.

L Genner, E. E., M.A. Io Crick Road, Oxford.

Giles, A. F., M.A. 23 Hope Terrace, Edinburgh.

Giles, P, Litt.D. LL.D. The Lodge, Emmanuel College, Cambridge.

Gilson, J. P, M.A. British Museum, W.C.1

Giveen, R. L. Colet Court, Hammersmith Road, $W$.

L Gooch, G. P, M.A. South Villa, Campden Hill Road, W. ${ }^{8}$

Gordon, Miss M. L. II 2 Victoria Road, Exmouth.

Gordon-Smith, C. S. Nutburst, Ditchling, Sussex.

Graham, Miss W. M. Old Bury Hall, Ightham, Kent.

Gray, Rev. J. H, M.A. Queens' College, Cambridge.

Greenup, G. F., B.A. I 4 Cambridge Park, Durdbam Downs, Bristol.

Greenwood, J. A, LL.M. Funtington House, near Chichester.

Greenwood, L. H. G, M A. Emmanuel Co'lege, Cambridge.

Gregory, Miss A. M. 7 Lansdowne Road, Wolverbampton.

Grundy, Prof. W. W. 'Lerryn,' Rbiwbina, nr. Cardiff.

Gulbenkian, Nubar S. c/o C. S. Gulbenkian, Avenue.d'Iéna, Paris, XVI.

Haddon, G. M. M. Glaisdale, Somerset Way, Iver, Bucks.

Hadow, Sir W. H, C.B.E. M.A. D.Mus. The University, Sheffeld.

H.ll, Miss M. I, M.A. Baldock, Herts.

H.ll. Miss W. I.. 4 Homefield Road, Bromley, Kent.

Hallam, G. H., M.A. S. Antonio, Tivoli (Roma), Italy.

Halstead, Major H. Duckworth, I 7 Brownsillle Road, Heaton Moor, Stockport.

Hanby, Miss K. M. Boulevard Secondary School, Hull.

Hannah, Ian C., F.S.A. The Whim, Lamancha, Peebiesshire.

Hardie, R. S. Io Greycoat Gardens, S.W.1

Hare, W. L. 3 Grav's Inn Place, W.C. ${ }^{1}$

L Harrison, E, M.A. Trinity College, Cambridge.

Hart, Percival. Grove Lodge, Highgate, $N$.

Hastings, G. 17 Welbury Drive, Bradford.

Hawkes, Christopher, B.A. British Museum, W.C.: 
Heath, C. H, M.A. 224 Hagley Road, Birmıngham.

Hedley, W. Percy. Gresbam House, Corbridge-on-Tyne.

L Heitland, W. E, M.A. . Carmefield, Wordsworth Grove, Cambridge.

Hemp, Wilfrid J, F.S.A. Minsbull, High Wycombe.

Henderson, Thomas. Educational Institute of Scotland, 47 Moray Place, Edinburgh.

Henry, Prof. R. M, M.A. Queen's University, Belfast.

Herbert, Rev. George. 6i Preston Rnad, Brighton.

Hignett, Charles, M.A. Hertford College, Oxford.

Hill, Mrs. B. H. 9 Plutarch Street, Athens, Greece.

Hill, G. F, M.A, LL.D., Litt.D., F.B.A. British Museum, W.C. ${ }^{1}$

Hill, Miss M. V, M.A. Uplands School, Archery Road, St. Leonards-on-Sea.

Hinks, Roger. British Museum, London, W.C. ${ }^{1}$

Hiorns, F. R, F.R.I.B.A. I88a, Adeiaide Road, St. Fobn's Wood, London, N.W."

Hirst. Prof. G. M. Barnard College, Columbia University, Newo York City, U.S.A.

Hirtzel, Miss Ruth. 47 Palace Court, $W .^{2}$

Hodge, Harold, M.A. 9 Higbbury Place, $N .^{5}$

L Hodgkin, R. H, M.A. 20 Bradmore Road, Oxford.

Hodgkinson, H. R. The Turret House, Hampton Lovett, Droitwich.

Hogan, Rev. W. K. P. Horsford Vicarage, Norwich.

Holden, Miss. 54 Piazza di Spagna, Rome. Italy.

Holland Martin, R., C.B., F.S.A. 46 Thurloe Square, S.W. ${ }^{7}$

Holmes. T. Rice, D.Litt., I.itt.D., F.B.A. I Akeburst Stret, Roebampton, $S . / I$

L Holroyd, Michael, M.A. Brasenose College, Oxford.

Hollis, Rev. A. M. Hertford College, Oxford.

Home. Major Gordon. I King's Bench Walk, E.C. ${ }^{4}$

Hopkins, G. B. I, M.A. Orley Farm Scbool, Harrow.

Hopkinson, Rev. Canon J. H., M.A. The Abbey, Carlisle.

Hurn, Theodore, M.A. The Grammar Scbool, Stockport.

I lornsby, W. Saltburn-by-the-Sea.

L Hose, H. F. Dulwich College, S.E. ${ }^{21}$

Hoskyns, Frances, Lady. 39 Sloane Gardens, S.W. ${ }^{1}$

Hotson, J. E. B. c/o Messrs. Grindlay \& Co, P.O., Box 93, Bombay, India.

Housman, Prof. A. E., M.A. Trinity College, Cambridge.

L Howard, E. C. Hotel Beau-Séjour, Mustapha, Algiers.

Hoyle, Miss A. M., B.A. 56 Carlton Road, Boston, Lincs.

Hulbert-Powell, Rev. C. Lacy. Burrell'; Field, Cambridge.

Hunt, James. Rydal School, Colwyn Bay.

Hunter Blair, C. H, M.A.. F.S.A. 57 Higbbury, Newcastle-upon-Tyne.

Hurst, W. T., M.A. 40 Chatel Street, Hyde, Cheshire.

L Hyde, J. H. 67 Boulevard Lannes, Paris, France.

Irons, Miss C. M., M.A. Aynhoe, Ashleigh Road, Horsham, Sussex.

Jarrett, E. A. Architectural Assnciation, 34 Bedford Square, W.C. ${ }^{1}$

James, Rev. J. W, B.D. Portskerwett, Mun.

Jardine, Miss Lindsay. 26 Murrayfield Road. Edinhurgh.

Jelf, Charles R. Trapstile, Lustleigh, Devon.

Jelf, E. A. St. Mary's Cottage, Waxwell Lane, Pinner.

Jenkin, Miss I. A. Wynberg, Cbestnut Avenue, Soutbborough, Tunbridge Wells

L Jex. Blake, Miss K. + Airlie Gardens, Campden Hill Road, $W .{ }^{8}$

Johnson, Miss E. I. 20 College Drive, Whalley Range, Manchester.

Johnson, Miss H. T. Ellesmere Lodge, The Park, Cheltenbam.

johnson, Miss Lorna A. Woodleigh, Altrincham.

Jolliffe, Prof. R. O. Queen's University, Kingston, Ontario.

Jones, H. L, M.A. Willaston School, Nantwich. 
xviii

Jones, Principal H. Stuart, D.Litt. F.B.A. University College of Wales, Aberystwyth.

Jones, Surgeon Captain K. H., R.N., M.B., F.Z.S. The Manor House, St. Stephen's, Canterbury.

L Jones, Ronald P. I 3 Hornton Street, S.W. ${ }^{8}$

Judson, William. Dean Close School, Cbeltenham.

Keiller, A. 4 Charles Street, Berkeley Square, $W .{ }^{1}$

Kelland, G. T., B.A. 5 Carling ford Road, Hampstead, N.W. ${ }^{3}$

Kendall, Miss O. P. University College, Gower Street, W.C. ${ }^{1}$

Kenyon, Sir Frederic, G.B.E., K.C.B., D.Litt., Litt.D., LL.D.,F.B.A. British Museun, W.C. ${ }^{1}$

Kerr, G. W. 7 Walsingham Avenue, West Didsbury, Manchester.

Kirk, J. L, B.A., M.B., B.Sc. Houndgate, Pickering, Yorks.

Klein, Walter G, F.S A. 7 Eldon Road, N.W. ${ }^{3}$

Knapton, Rev. A. J. The Vicarage, Clee St. Margaret, Craven Arms, Sbropshire.

Knight, W. F. J. Bloxbam School, nr. Banbury, Oxford.

Knowles, W. H, F.S.A., F.R.I.B.A. Well Close, Lansdown Parade, Cbeltenham.

Knox, Rev. W. L. The Oratory House, Lady Margaret Road, Cambridge.

Langford, F. C. G., M.A. 35 Alley Park, Dulwich, S.E. ${ }^{21}$

Last, H. M, M.A. St. Fobn's College, Oxford.

L Laurand, Dr. L. 37 Boulevard de Tours, Laval (Mayenne), France.

Laurie, G. E. Roval Academical Institute, Belfast.

Laver, P. G., F.R.C.S., F.S.A. Broomfield, Ardleigh, Essex.

Lawrence, Sir A. 49 Marsham Street, S.W. ${ }^{1}$

Lawson, P. H., F.S.A. 2 Pepper Street, Chester.

Leeper, Alexander, LL.D. St. Audoen's, Kensington Road, S. Yarra, Melbourne, Australia.

Leishman, Miss E. M. R. $4 \mathbf{I}$ Campden Hill Road, $W .{ }^{8}$

Letts, M. H. I. Io Bartlett's Buildings, Holborn Circus, E.C. ${ }^{4}$

Lewer, H. W, F.S.A. Priors, Loughton, Essex.

Lewis, Miss A. K. Brighton and Hove High Scbool, Brighton.

L Lewis, J. Spedan clo Peter Fones, Ltd., Sloane Square, S.W. ${ }^{1}$

Lewis, Miss M. E. 6 Hillside, S.W. ${ }^{19}$

Linaker, R. Hyde, F.S.A. Rock Costage, Frodsham.

Lindley, Miss. 74 Shooter's Hill Road, Blackbeath, S.E. ${ }^{3}$

Livett, Rev. Canon G. M, F.S.A. Belmont, Wray Park Road, Reigate, Surrey.

Lloyd, Prof. J. E, M.A., D.Litt., F.S.A. Gwaen Deg, Bangor.

L Loeb, James. Hocbried, Murnau/Staffelsee, Bavaria.

Longbotham, G. F. 378 Linthorpe Road, Middlesbrough.

Longden, R. P., M.A. Christ Cburch, Oxford.

Low, Miss Janet I. Blebo, Cupar, Fife.

Lowndes, Miss. c/o Mr. James Bain, I4 King William Street. Strand, W.C."

Lowther, A. W. G. The Old Quarry, Ashtead, Surrey.

McCulloch, Miss J. G., M.A. 2 Westbourne Terrace, Ibrox, Glasgow.

McCulloch, W. Simon Langton Schools, Canterbury.

L Macdonald, Sir George, K.C.B., D.Litt., LL.D., F.B.A. I7 Learmonth Gardens, Edinburgh.

Macdonald, Mrs. J. G. 3 Hope Street, St. Andrews, Scotland.

L Macdonald, Miss L, M.A. 47 Seymour Street, Portman Square, $W .{ }^{1}$

McDonell, H. C. M, M.A. Twovford Scbool, Winchester. 
Macdonell, The Hon. Sir P. J. Supreme Court, Trinidad.

McElderry, Prof. R. Knox. Glenside, Sans Souci Park, Belfast.

McEvoy, Rev. C. 'Alesia,' Parkside Drive, Watford, Herts.

MacGregor, Rev. W, M.A., F.S.A. Boleball Manor House, Tamworth.

McIntyrc, James. West View, Bishop Auckland.

McLellan, R. D. go Stanstead Road, Hertford.

Mackail, J. W, M.A., LL.D., F.B.A. 6 Pembroke Gardens, $W$.

L Mackey, Rev. Father Peter Paul, O.P. Collegio Angelico, 5 Via San Vitale, Rome, Italy.

L Macmillan, Sir Frederick. 22 Devonshire Place, $W .{ }^{1}$

Macmillan, George A, D.Litt. 27 Queen's Gate Gardens, S.W.'

Macmillan, W. E. F. 42 Cnslow Square, S. $W . ?$

McMurtrie, Miss B. S. B. Craigrowan, Kilmacolm, Renfrewshire.

McNabb, Miss E. R. 62 Great George Street, Hillhead, Glasgow, $W .{ }^{2}$

L Magic, Prof. David. Ior Library Place, Princeton, New Fersey, U.S.A.

Magoffin, Prof. R. V. D. New York University, University Heights, New York City, U.S.A

Magrath, Rev. J. R, D.D. Provost's Lodgings, Queen's College, Oxford.

Makin, Miss Ena, M.A.

Manion, Rev. B., M.A. Cotton College, North Staffs.

L Mardon, A., M.A. The Rice Ivistitute, Houston, Texas, U.S.A.

Marks, Geoffrey, C.B.E. Union Club, Io Carlton House Terrace, S.W. ${ }^{1}$

Marriage, Miss E. G. 62 Pont Street, S.W. ${ }^{1}$.

Marshall, Miss A. M. C. 3 Roedean School, Brigbton.

Marshall, D. W. H., M.A., I.L.B., B.Litt. University of Manitubn, Winnipeg, Canada.

Marston, Sir Charles, J.P. Afcot, Compton, Wolverhampton.

Martin, Miss A. P., M.A. 24 Murray House, Vaudon Street, S.W. ${ }^{1}$

Martin, Alan R. I8 Kidbrook Park Road, S.E. ${ }^{3}$

Matheson, P. E., M.A. 3 Brookside, Headington, Oxford.

Mattingly, H, M.A. British Museum, W.C. ${ }^{1}$

Maufe, F. B. I5 Hanover House, Regent's Park, N...W 8

May, Thomas, M.A., F.S.A., F.S.A.Scot., F.E.T. Loxley Lodge, Loxley Road, Stratfordon-Avon.

Meade, The Hon. Mrs. Edward.

Meiggs, R., M.A. Keble College, Oxford.

Meiklejohn, K. W. St. Bees School, Cumberland.

Michie, Prof. J. L, M.A. The University of Queensland, Brisbane, Austraiia.

Mildmay, Dr. E. St. John. Via Telesib 19, Milan.

Miles, Sir J. C, M.A. Merton College, Oxjord.

Miller, S. N, M.A. I5 Doune Terrace, Kelvinside N., Glasgow.

Millington, Miss M. V. 30 Peak Hill, Sydenbam, S.E. ${ }^{26}$

Mills, Miss Dorothy. The Brearley Scbool, 610 East 83rd Street, New York, U.S.A.

Mills, T. R. University College, Dundee.

Milne, J. G., M.A., D.Litt. 20 Bardwell Road, Oxford.

Minns, Prof. E. H, Litt.D. Pembroke College, Cambridge.

Mond, Mrs. Angela. 22 Hyde Park Square, $W .{ }^{2}$

Montague, Lt.-Col. L. A. D., J.P., F.R.N.S. Penton, Crediton, N. Devon.

Moon, Miss E. M. $6_{3}$ Oakwood Court, Melbury Road, Kensington, W. ${ }^{4}$

Morcom, Col. Reginald K. The Clockbouse, Bromsgrove.

Morris, W. J, B.A. 23 Birchgrove Street, Portb, Rbondda.

Mothersole, Miss Jessie. Pilgrim's Peace House, Hampstead, N.W. ${ }^{3}$

Mozley, J. H., M.A. Kentons, Haslemere, Surrey.

Muirhead, A. M. 83 The Limes Avenue, N. Southgate, $N .{ }^{11}$

Muirhead, J. Spencer, LL.B., D.S.O., M.C. 205 St. Vincent Street, Glasgow.

Munro, Miss Isabel, B.A. Lincoln College, Oxford.

Munro, J. A. R, M.A. Lincoln College, Oxford.

Murray, Prof. G. G. A., D.Litt., LL.D., F.B.A. Boar's Hill, Oxford. 
L Myres, Prof. J. L, M.A.. D.Litt., F.S.A. The Copse, Hinksey Hill, Oxford.

L Myres, J. N. L., B.A. Christ Church, Oxford.

Nairn, Rev. J. A. Stubbings Vicarage, Maidenbead.

Nairne, Prof., D.D. Jesus College, Cambridge.

Nash, A. B. 36 Priory Road, Hornsey, N. ${ }^{8}$

Nash-Williams, A. H. "Olivone," 67 Soutbwood Road, Ramsgate.

Nash-Williams, V. E. The National Museum of Wales, Cardiff.

Newbold, W., O.B.E., H.M.I. clo Board of Education, Whiteball, S.W.'

Newman, Miss D. H. 170 Holland Road, $W .{ }^{14}$

Nock, Prof. A. D., M.A. Widener Library, Harvard College, Cambridge, Mass. U.S.A.

Norman, Philip, LL.D., F.S.A. 45 Evelyn Gardens, S.W. ${ }^{7}$

Norris, Miss M. E. County High School for Girls, Church Hill, Walthamstow, E. ${ }^{7}$

Norwood, Prof. G. Universitv College, Toronto, 5 Canada.

Oakeshott, W. F. 54 Prince of Wales Mansions, Battersea, S.W.11

Oakley, H. C., M.A. 22 Ouseley Road, Balham, S.W.12

Ogle, Christopher. Browne's Lodge, Reigate.

Oldfield, Rev. W. J, D.D. Prcb. of Lincoln, White Lodge, Abingdon.

Oliphant, Prof. S. G, Ph.D. 520 Stewart Avenue, Grove City, Pennsylvania, U.S A.

Oliver, J. R., M.D. The Latrobe Apartments, Read and Charles Streets, Baltimore, U.S.A.

Ormerod, Prof. H. A., M.C., M.A. 25a Falkner Square, Liverpool.

Oswald, Felix, D.Sc., F.G.S., F.S.A. H.M. Probate Registry, Nottinghiam.

Owen, A. S, M.A. Keble College, Oxford.

Owen, E. Bisbop's University, Lennoxille, Quebec, Canada.

Owen-Mackenzie, Lady, 6 Chesham Street, S.W.1

Page, T. E, Litt.D. Charterbouse, Godalming.

Palmer, A. E. Penryn, Temple Road, Stcwmarket.

Pantin, W. E. P., M.A. 17 Dewburst Road, West Kensington, W. ${ }^{14}$

Parish, A. Brockbolt, Otford, nr. Sevenoaks, Kent.

Parker, H. M. D, M.A. Magdalen College, Oxford.

Parker, Colonel J. W. R, C.B., D.L., F.S.A. Browsbolme Hall, Clitheroc.

Parker, Dr. W. Rushton. Royal Institution, Albemarle Street, $W{ }^{1}$

Passmore, A. D. Callar House, Wanboroagh, Wilts.

Paterson, Prof. A. C. Auckland University College, Auckland, New 7ealand.

Payne, Major A. A. Laporte. 47 Eton Court, Eton Avenue, N.W. ${ }^{3}$

Payne, B. Io3 Hyde Park Road, Leeds.

Peachey, Geoffrey E. Pook's Hill, Hove, Sussex.

Pearce, J. W. E, M.A. Io Cromwell Place, S.W.'

Pearson, Prof. A. C, Litt.D. Trinity College, Cambridge.

Pearson, Miss E. R, M.A. \& Woburn Mansions, Francis Street, W.C. ${ }^{1}$

Pease, Mrs. Nether Grange, Alnmouth.

Penoyre, John, C.B.E. M.A. 8 King's Bench Walk, Inner Temple, E.C. ${ }^{4}$

Penrose, Dame Emily, M.A., D.C.L. I4a Belsize Park Gardens, N.W. ${ }^{3}$

L Perram, Charles, M.D. Norland Court, 82 Gloucester Place, Portmun Square, W. ${ }^{1}$

Perry, F. T. Beech Close, Car Colston, Notts.

Petch, J. A, M.A. The University, Mancbester.

Pickard-Cambridge, Prof. A. W, M.A. The University, Edinburgh.

Pickering, A. J., F.G.S. Abbeycraig, Hinckley, Leics.

Pickstone, Miss J. North Middlesex School for Girls, Enfield Chase, Middlesex.

Pierce, S. R. 39 St. James' Strett, Bedford Row, W.C. ${ }^{1}$ 
L Piatnauer, Maurice, M.A. Brasenose College, Oxford.

L Plunkett, Count G. N., T.D. 40 Elgin Road, Dublin.

Pocock, L. G. 26 Stanbope Road, Highgate, N. ${ }^{6}$

Pollock, The Rt. Hon. Sir F., Bart., M.A., LL.D., D.C.L., F.B.A., F.S.A. 21 Hyde Park Place, $W .{ }^{2}$

L Pope, Rev. J. O'Fallon, S.J. Manresa House, Roebampton, S.W. ${ }^{15}$

Porter, W. H. Lebenagh House, Cork.

Pratt, E. A. 4 Adelphi Terrace, W.C. ${ }^{2}$

Pratt, F. J. Tidd. 22 Balderton Gate, Newark.

Prescott, H. M. 137 High Lever Road, W. ${ }^{10}$

Preston, A. W. Bishop's College, Lennoxville, Quebec, Canada.

L Preston, Colonel J. E. c/o Grindlay छ゙ Co., 54 Parliament Street, S.W. ${ }^{1}$

Price, Stanlev, M.A. Glorishears, Rigtonbill Estate, East Keswick, Leeds.

Prosser, W. B. Ardenlee, Maidstone.

Pryce, T. Davies, M.R.C.S, F.S.A. Bramber, Fletcher Road, Horsell, Surrey.

Purser, Louis C., D.I.itt., Litt.D., F.B.A. $8 \mathrm{~W}$ aterloo Road, Dutlin.

Radcliffe, William. Beech Court, Upper Deal, Kent

Radford, C. A. Ralegh, F.S.A. Bradninch Manor, Devon.

Ramsay, A. B., M.A. Magdalene College, Cambridge.

Ramsay, Miss A. M., M.A. Lady Margaret Hall, Oxford.

L Ramsay, Sir W. M., D.C.L., Litt. D., LL.D., D.D., F.B.A. 55 Soutbbourne, Overcliff, Bournemouth.

L Randall, H. J., LL.B. Erw Graig, Bridgend, Glamorgan.

Randall-MacIver, D., M.A., D.Sc. 25 Corso d'ltalia, Roma, Italy.

Rann-Kennedy, W. J, M.A. F.R.G.S. 6 Castle Hill Road, Dover, Kent.

Reade, W. H. V, M.A. Keble College, Oxford.

Reckitt, G. F. 10 Downleaze, Sneyd Park, Bristol.

Record, S. P. M.A. Taunton School, Taunton.

Reed, A. E. Grammar Scbool, Brigg, Lincs.

Reed, Miss G. N. Girls' Grammar School, $W_{\text {atforl }}$.

Richards, Frank, M.A. 54 Mapperley Road, Nottingham.

Richards, F. L, Assoc.M.Inst.C.E. Penryn, Kingsway, Woking, Surrey.

Richards, Rev. J. F. Sonth Luffenbam Rectory, Stamford, Lincs.

Richardson, Miss A. W. Westfield College, Hampstead, N.W.

Richardson, L. J. D., M.A. Fernshaw, Hilary Road, Llanishen, Cardiff.

Richmond, Ian A., M.A. Buckiey Hill House, Milnrow, Rocbdale.

Richmond, J., B.A. Box I487, Stn. C., Los Angeles, California.

Richmond, Prof. O. L, M.A. 5 Belford Place, Edinburgh.

Rickard, T. A. 250 Tunnel Road, Berkeley, California.

Roberts, Miss D. G., M.A. County Girls' Scbool, Bisbop Auckland, Durbam.

Roberts, J. Slingsby, 3 Powis Villas, Brigbton.

Roberts, Miss M. D: Ingleburst, Huddersfield Road, Halifax, Yorks.

Robertson, Prof. D. S, M.A. Trinity College, Cambridge.

Robertson, Prof. L. University of British Columbia, Vancouver, B.C.

Robinson, Prof. C. A., Jr. 63 Angell Street, Prozidence, R.I., U.S.A.

L Robinson, E. S. G., M.A. 23 Upper Phillimore Gardens, $W .^{8}$

Rodd, The Rt. Hon. Sir Rennell, G.C.M.G.,G.C.V.O.,C.B. 25 Cavendish Square, $W^{\prime} 1$

Roeber, A. D. Eckington Villa, London Road, Reigate, Surrey.

Roos, Dr. A. G. 7 Waterloolaan, Groningen, Holland.

Rose, Prof. H. J., M.A. Edgecliff East, The Scores, St. Andrews, Fife.

Rossiter, Miss G. M, B.A. The High School, Beverley, Yorks.

Rothwell, Miss H. L. Moorelands, Malvern Wells.

Rouse, W. H. D., M.A., Litt.D. H iston Manor, Cambridge.

Rowland, L. G. Thwaitefield, Goatbland, rorks. 
Rushforth. G. McN, M.A., F.S.A. Riddlesden, Malvern Wells.

Russell, Miss Agnes. Maltese Lodge, Chelmsford.

Ryott, H. A. Leathley, Nr. Otley, Yorks.

Salisbury, F. S., M.A. Dorset House, Asbford, Middlesex.

Salmon. E. T.. B.A. Loughton House, Lougtton, Essex.

Sands, P. C., M.A. Pocklington School, East Yorks.

Saunders, G. F., M.A. clo Mrs. Kirby, Manor House, Redbridge, Soutlampton.

Sayce, Prof. A. H, D.Litt., Litt.D., LL.D., D.D. 8 Chalmers Crescint, Edinlurgh.

L Scarth, Miss W. G. $97^{10}$ Cadogan Gardens, S.IW. ${ }^{3}$

Schede, Prof. Konstantinopel, Pera, A.P.I42, Constantinople, Turkey.

Scott, J. E, M.C., M.A. Gonville and Caius College, Cambridge.

Scullard, H. H. 52 Foscote Road, Hendon, N.W. ${ }^{4}$

Seltman, C. T., M.A., F.R.N.S. 39 Barton Road, Cambridge.

Semple, Prof. Patrick, M.A. Earlsfoot Terrace, University College, Dublin.

Seymour, P. A., M.A. Fesus College, Oxford.

Sharpe, Sir Montagu, K.C. D.L. Brent Lodge, Hanwell, $W$.

Shaw, Rev. J. Tunnicliffe. 47 Grange Avenue, Chapeltown Road, Leeds.

Shaw, R. C., F.R.C.S., L.R.C.P. I68 Tulketh Road, Ashton, Preston, Lancs.

Sheepshanks, A. C. Eton College, $W$ indsor.

Sheppard, J. T, M.A. Litt.D. King's College, Cambridge.

Sherman, Prof. C. P., D.C.L., LL.D. 3430 Thirty-fourth Street, N.W., Washington, D.C., U.S.A.

Sherwin, G. A. Bramford, Cypress Road, Newport, I. of Wight.

Shields, Mrs. van Allen. 9 rue Spontini, Paris $\left(x v i i^{e}\right)$, France.

Shove, Miss E. 330 Upper Richmond Road, Putney, S.W..$^{15}$

Simpson, F. G., M.A., Hon.F.S.A. (Scot.). 45 Fern Avenue, Newcastle-on-Tyne.

Sisson, M. A. Hucclecote, Near Gloucester.

Skeat, T. C. Romelandfield, Ramsbury Road, St. Albans.

Sleeman, J. H, M.A. Malabide, Englefield Green, Surrey.

Sloane, Miss E. J. 8 University Road, Leicester.

Smith, A. H., C.B., F.S.A. 2 Balfour Road, Weybridge, Surrey.

Smith, G, M.A. Dulwich College, S.E. ${ }^{21}$

Smith, G. E. Lendrick Lodge, Stokes Hill, Wateriooville, Hants.

Snyder, Prof. G. A. S. Allard Pierson Stichting, Weesperzyde, 33, Amsterdam, Holland. Souter, Prof. A., M.A., D.Litt., D.D., F.B.A. 9 Coliege Bounds, Aberdeen.

Spiers, Henry, M.D., F.R.C.S.Ed. St. Dunstan's House, Melrose, Scotland.

Staines, E. A. Controller of Posts and Telegrapbs, Selangor \& Pebang, G.P.O. KualaLumpur, Fed. Malay States.

Stannard, Harold, M.A. 55 Russell Square, W.C. ${ }^{1}$

Stevens, G. C., B.A. The School, Sbrewsbury.

Stevens, C. E., B.A. 3 I St. Aldate's, Oxford

Stevenson, G. H, M.A. University College, Oxford.

Stewart, Prof. H. The University, Leeds.

Stewart, Prof. J. A, LL.D. Cbrist Church, Oxford.

Stinton, T., M.A. The High School, Newcastle, Staffs.

Strangeways, L. R, M.A. The Grammar School, Bury, Lancs.

L Streatfield, Mrs. 22 Park Street, Mayfair, $W .{ }^{1}$

Strong, Mrs. S. Arthur, LL.D., Litt.D., F.S.A. 35 Via Balbo, Rome, Italy.

Strudwick, Miss Ethel, M.A. St. Paul's Girls' School, Brook Green, Hammersmith, $W{ }^{6}$

L Sutcliffe, Rev. E. F., S.J. Hevthrop Hill, Chipping Norton, Oxun

Swallow, Rev. Canon. Chigwell Scbool, Essex.

Swinburne, Lady. Capheaton, Newcastle-on-Tyne.

Swithinbank, B. W., I.C.S. clo Thomas Cuok So Son, Rangoon, Burma.

L Syme, Ronald, B.A. Trinty College, Oxford.

Symonds, Rev. H. H, M.A. Io Riversdale Road, Aighurth, Liverpool. 
Tandy, F. 75 Palace Road, Tulse Hill, S.W.z

Tarn, W. W. Lancaster Gate Hote?, W. ${ }^{2}$

Taylor, Sir Andrew T., Knt., F.S.A. Drummond Lodge, 2 I Lyndhurst Road, Hampstead, $N . W .{ }^{3}$

Taylor, H. M. 67 Monk Road, Ward End, Birmingbam.

Taylor, J. E, M.A., B. Litt. Tonbridre Scbool, Tonbridge.

Taylor, Prof. I.. R. Latw Buildings, Brwn Mawr College, Pa., U.S.A.

Taylor, Miss M. V., M.A., F.S.A. 45 Woodstock Road, Oxford.

Taylor, W. G. 34 Higb Oaks Road, Welwyn Garden City, Ilerts.

Thomas, G. P. F. St. Edzwards, Broadstairs, Kent.

Thompson, J., M.A. 28, St. Ledgers Road, Bournemouth.

L Tod, Marcus N, M.A. Oriel College, Oxford.

Towers, R. M, M.A. The College, Cheltenbam.

Townsend, Prof. P. W. I 200 E. First Street, Bloonitington, Ind., U.S.A.

Toynbee, Prof. Arnold J, M.A 3 Melina Place, St. 'Fobn's Wood, N.W. ${ }^{8}$

Toynbee, Miss Jocelyn, M.A. Newnbam College, Cambridge.

Turnbull, G. L, M.A. The Academy, Dingwall, Scotland.

L Turner, Prof. C. H, D.Litt. F.B.A. Magdalen College, Oxford.

Urquhart, F. F, M.A. Balliol College, Oxford.

Van Buren, Prof. A. W. American Academy, Porta S. Pancrazio, Rome, Italy.

Van Essen, C. C. Museum, Carnegie Lane, Hague, Holland.

Vaughan, W. W, M.A. The Lodge, Rugby School, Rugby.

Wackernagel, Prof. J. 93 Gartenstrasse, Basle, Switzerland.

Walker, Rev. F. G. The Rectory, Uptnn Lovel, Wilts.

Walker, R. H. Hillbrow 4, Rugby.

Walker, W. G, M.A. Crosby House, Oundle, Ptterborough.

Walker, W. W, M.A. Io Queen Anne Terrace, Cambridge.

Wallace, W. Reeve. 4 Waverton Street, $W .{ }^{1}$

Wallis, Mrs. A. Morland, Darlington.

Wallis, D. E., B.A. 25 Sutberland Road, West Ealing, $W .{ }^{13}$

Walters, H. B, O.B.E., M.A., F.S.A. British Museum, W.C.'

Ward, W. W. Merchants' Hall, Bristol.

Warren, Miss. 5I Morwen Avenue, Mansfield Wudbusise, Notts.

L Watson, Rev. Prof. E. W, D.D. Cbrist Cburcb, Oxford.

Watson, J. M. H. Fort-les-Bains, Amélie-les-Bains, Pyrénées Orientales, Franse.

Watson, W. Crum. Northtield, Balcrno, Midlothian.

Weavers, Miss E. M, B.A. 2 Goodroyn Avenue, Mill Hill, N.W. ${ }^{7}$

Webb, Prof. Clement C. J., M.A., F.B.A. Walnut Tree House, Marston, Nr. Oxford.

Weber, F. Parkes, M.D. F.S.A. I 3 Harley Street, $W .{ }^{1}$

Webster, Miss M. G. Alexandra College, Westcliff-on-Sea.

Webster, T. B. I., Christ Church, Oxford

Wedd, N, M.A. King's Coilege, Cambridge.

Weddell, A. W. Virginia Flouse, Windsor Farme, Richmond, Virginia, U.S.A.

Weeck, W. N., M.A. Greenfields, The Park, Cheltenbam.

Weekes, C. A. 3I Cannon Hill, London, N.W. ${ }^{6}$

L Westland, F. C., Lt.-Col. R.E. c/o Lloyds Bank, Cox's and King's Branch, 6 Pall Mall, $S . W .1$

Whatley, N, M.A. Clifton Coillege, Bristol.

Wheeler, R.E. Mortimer, D.Litt., F.S.A. London Museum, Lancaster House, St. Fames's, S.W. 1 
xxiv

Whitehead, G. O. 26 Pelham Crescent, S.W. ${ }^{7}$

Whiteley, S. University College, Colombo, Ceylon.

L Whittick, G. Clement, M.A. Armstrong College, Newcastle-on-Tyne.

Whitney, Rev. Prof. J. P, D.D. Emmanuel College, Cambridge.

Wilde, Mrs. II Stanley Crescent, $W .{ }^{11}$

Wilkinson, M. Bow, Durb.am.

L Willans, J. B. Dolforgan, Kerry, Montgomeryshire.

Williams, Miss G. H. The College, Pontypool, Mon.

L Williams, R. M.C. M.A. The Grammar School, Cosobridge, Clam.

Williams, Rev. W. Coleman. Caerwent Vicarage, Caerwent, nr. Chepstow.

Williams, Walter N, M.A, LL.B. Selwyn College, Cambridge.

Williamson, Prof. H., M.A. 34 Soutbway, N.W.11

Wilson, Mrs. G. M. Risebolme, Lincoln.

Wilson, Major H. C. B. Crofton Hall, Crofton, Wakefield.

Winbolt, S. E., M.A. Aclea, Worthing Road, Horsham.

Wood, H. McKinnon, B.A.

L Wood, J. C, B.A. 4 Carlisle Place, Bradford.

Woodard, E. A. The Caffinites, Durbam School, Durbam.

Woodward, Miss A., M.A. Royal Holloway College, Englefield Green, Surney.

Woodward, A. M., M.A. 8 Gloucester $W$ alk, $W .8$

Woodward, IV. H, M.A. 39 Harley House, N.W. ${ }^{1}$

Woolley, C. L., M.A.

Woolley, Ernest, F.S.A. Collingriorth, Lemsford Road. St. Albans.

L Worley, Miss M. L., M.A. Quarry Field Cottage, London Road, Headington, Oxford.

IVright, W. E. 14 Wilbury Road, Hove, Sussex.

Wynne-Jones, Mrs. C. Thombill, Stalbridge, Dorset.

Yapp, Miss M. G. The Friends' Scbool, Saffron Walden, Essex.

Yorath, Miss E. L. 9 Elliston Terrace, Carmarthen, S. Wales.

L Young, Miss A. M. Blackbeath High School, Wemyss Road, Blackbeath.

Young, G. M. All Souls College, Oxford.

Young, K. D, F.R.I.B.A. I7 Southampton Street, W.C. ${ }^{1}$

\section{STUDENT-ASSOCIATES.}

Alcock, Philip. Emmanuel College, Cambridge.

Allan, D. J. I3 West Parade, Norwich.

Anderson, Andrew C. 14 Trinity College, Dublin.

Barnes, Miss M. A. Earnseat, Arnside, Westmorland.

Behrens, W. E. B. Surnton Grange, Malton, Yorks.

Charlton, J. 32 The Avenue, Gateshead, Co. Durbam.

Compton, E. G. 68 Ennismore Gardens, S.W.'

Granville, B. 25 Bloomfield Terrace, S.W. ${ }^{1}$

Griffith, P. T. Worcester College, Oxford.

Hamilton, J. A. S. Trinity College, Oxford.

Hearnshaw, L. S. 28 Spencer Hill, Wimbledon, S.W. ${ }^{19}$

Helmore, J. R. C. I9 Pboenix Lodge, Brook Green, $W .^{8}$

Hollis, H. N. East Liberty, Wells, Somerset.

How, F. W. 21 Merton Street, Oxford.

King, J. B. Newonbam College, Cambridge.

Lake, Phyllis. I 2 Southfield Road, Norwich.

Linton, S. 2 Io Clive Road, S.E. ${ }^{21}$

Long, R. S. 3 Richmond Terrace, Cambridge.

Lucas, Diana. 50 Oakley Street, Chelsea, S.W. ${ }^{3}$

McCormick, Patrick. 339 Main Street, Rutherglen, Glasgow. 
Mott, Mrs. Ruth. Mancbester Univ. Settlement, Every Street, Ancoats, Manchester.

O'Neill, D. E. Innisfree, Balmoral, Belfast.

Padley, C. S. Io Canterbury Drive, Fulwood, Sheffield.

Page, D. L. The Gables, 108 Albert Road, Caversham, Reading.

Pearson, L. I. C. 45 Madeira Park, Tunbridge Wells.

Salt, H. R. M. Pembroke College, Oxford.

Skinner, K. A. Newnbam College, Cambridge.

Somerville, R. Lindrum, Queensferry Road, Dunfermline.

Sorroff, L. E. E. King's College Hostel, Vincent Square, S.W. ${ }^{1}$

Sparrow, John. All Souls College, Oxford.

Stevens, P. T. IIA Aigburtb Hall Road, Liverpool.

Sutherland, C. H. V. Christ Church, Oxford.

Wilson, F. H. 18 Parkfield Avenue, Bradford Moor, Bradford, Yorks.

Winnifrith, A. J. D. Restoration House, Rocbester.

Wolferston, D. P. Forwood, Mincbinbampton, Stroud, Gluucs.

\section{LIBRARIES SUPPLIED UNDER COPYRIGHT AC'T.}

Cambridge: The University Library.

OXFord: Th: Bodleian Library.

Dublin : The Library of Trinity College.

Edinburch : The Advocate;' Library.

Aberystwyth : The National Library of Wales.

London: The British Museum, Copyright Office, W.C. ${ }^{1}$

\section{SUBSCRIBING LIBRARIES AND INSTITUTIONS.}

\section{GREAT BRITAIN AND IRELAND.}

Aberdeen : The Library of the University, Aberdeen.

Aberystwytu : The Library of the Univers.ty College of Wales, Aberystwyth.

Aldenham: The Library of Aldenham School, Elstrec, Herts.

Ampleforth : The Library of Ampleforth Abbey, $r_{\text {ork. }}$

Bancor : The Library of University College of North Wales, Bangor.

Belfast : The Library of the Queen's University, Belfast.

Birmingham : Th: Birmingham Archacological Society, Birmingbam

Birmingham : The Central Free Library, Birmingbam.

Birmingham: The University Library, Birmingham.

Bradford : The Central Library, Bradford.

Brichton : The Public Library, Brigbton.

Bristol : The Library of the University, Bristol.

Cambridge : The Library of Emmanuel College, Cambridge.

Cambridge: The Library of King's College, Cambridge.

Cambringe: The Library of Newnham College, Cambridge.

Cambridge: The Library of St. John's College, Cambridge.

Cambridge: The Museum of Classical Archaeology, Cambridge.

Cambridge: Trinity College Library, Cambridge.

Cardiff: The Cuntraj Iibrary, Curdiff.

Cardiff: The National Museum of Wales, Cardiff.

Cardiff : The University College of South Wales, Cardiff.

Charterhouse: The Library of Charterhouse School, Godalming.

Cheltenham : I ne Library of the Ladies' College, Cbeltenbam.

Chester : Chester and N. Wales Archaeological Society, Chester.

Colchester: The Library of the Corporation Museum, Colchester. 
Derby : The Public Library, Derby.

Dublin : The King's Inns Library, Dublin.

DubLin : The National Library of Ireland, Dublin.

DunDEE: The Albert Institute and Victoria Galleries, Dundee.

Dunder: The Library of University College, Dundee.

Durham : The Library of the University, Durbam.

Edinburgh : The Public Library, Edinburgh.

Edinburgh: The Royal Scottish Museum, Edinburgh.

EdinBURGH : The Signet Library, Edinburgh.

EDINBURGH : The Library of the University, Edinburgh.

Englefield Green, Surrey: The Royal Holloway College, Englefield Green.

Eron: The Library of Eton College, Windsor.

Galway: The Library, University College, Galway.

GlasGow : The Library of Baillie's Institution, Glasgow.

GlasGow: The Mitchell Library, Glasgow.

Glascow: The Library of the University, Glasgow.

Gloucester: The Bristol and Gloucestershire Archaeological Society, The Public Library, Gloucester.

Hawarden : St. Deiniol's Library, Hazbarden.

Huddersfield : The Public Library, Huddersfield.

Hulr : The Central Public Library, Hull.

Huld: The Municipal Museum, Hull.

Huld : University College Library, Hull.

Leeds: The Library, Commercial Street, Leeds.

LeEDs: The Library of the University, Leeds.

Leicester: The Library of Wyggeston Boys' School, Leicester.

Liverpool: The Library of the University, Liverpool.

Liverpool: The Public Library, William Brown Street, Liverpool.

London: The Library of the Athenaeum Club, London.

London: The Department of British and Medieval Antiquities, British Museum, London.

London: The Department of Greek and Roman Antiquities, British Museum, London.

London: The Library of the Burlington Fine Arts Club, London.

Lonson: The Guildhall Library, London.

London: 'The Library of King's College, London.

London: The London Library, St. James's Square, London.

London: The Library of Merchant Taylors' School, S.E. ${ }^{1}$

London: The Library of the Oxford and Cambridge Club, London.

London: The Library of the Reform Club, London.

London: The Library of the Royal Anthropological Institute, London.

London: The Library of the Royal Geographical Society, London.

London: The Library of the Royal Institution, London.

London: The Library of the Royal Institute of British Architects, London.

London: The Library of Sion College, London.

London: The Library of the Victoria and Albert Museum, London.

London: Westfield College, Hampstead, London, N.W. ${ }^{3}$

London: The Library of the City of Westminster, London, S.W. ${ }^{1}$

London: The Library of Westminster School, London.

London: Dr. Williams's Library, London.

Manchester : The Library of the Grammat School, Manchester.

Manchester: The John Rylands Library, Manchester.

Manchester: The Library of the Victoria University, Mancbester.

Newcastle-upon-Tyne: The Library of Armstrong College, Newcastle-upon-Tyne.

Newcastle-upon-Tyne: The Literary and Philosophical Society, Nerocastle-upon-Tyne.

Newcastle-upon-Tyne: The Public Library, Newcastle-upon-Tyne.

Nottingham : The Library of University College, Nottingham.

OundeE: The Library of Oundle School, Oundle. 
Oxford: The Library of All Souls College, Oxford.

Oxford: The Library of the Ashmolean Museum, Oxford.

Oxford: The Library of Balliol College, Oxford.

Oxford: The Library of Brasenose College, Oxford.

OxFord: The Library of Christ Church, Oxford.

OXford : The Senior Library, Corpus Christi College, Oxford.

Oxford: The Library of Exeter College, Oxford.

OXford: The Library of Hertford College, Oxford.

Oxford: The Library of Jesus College, Oxford.

Oxford: The Library of Keble College, Oxford.

Oxford: The Library of Magdalen College, Oxford.

OXFORD : The Library of Manchester College, Oxford

OxFord: The Library of Merton College, Oxford.

OxFord: The Library of New College, Oxford.

Oxford : The Library of Oiiel College, Oxford.

OXFord: The Library of Somerville College, Oxford.

Oxford: The Library of St. John's College, Oxford.

OXFORD: The Library of Trinity College, Oxford.

Oxrord: The Library of University College, Oxford.

OxFord: The Library of the Union Society, Oxford.

Plymouth: The Free Public Library, Plymouth.

READING : The Library of the University, Reading

RugBY : The Arnold Library, Rugby School, Rugby.

St. Andrews: The Library of the University, St, Anarews.

Sheffield: The Free Public Library, Sbeffield.

Sheffield : The Library of the University, Sheffeld.

Southampton: Ordnance Survey, Soutbampton.

Southampton: University College Library, Southampton.

Soutuend-on-Sea: The Central Public Library, Southend-on-Sea.

Streatham Hile: The Library of the Streatham Hill High School, Streatham Hili.

Swansea: The Library of University College, Swansea.

UPPINGHam: The Library of Uppingham School, Uppingham.

Wantage: St. Mary's School, Wantage, Berks.

Warrington: The Director of the Corporation Museum, Warrington.

Windsor: The Library of Beaumont Colleg, Old Windsor.

York: The Yorkshire Philosophical Society, The Museum, rork.

York: The Yorkshire Museum, York.

\section{BRITISH DOMINIONS.}

Adelaide, S. Australia: The Public Library, Adelaide.

Adelaide, S. Australia: The Library of the University, Adelarde.

Brisbane, Quennsland: The Library of the University of Queensland, Brisbane.

Cape Town, S. Africa: The South African Public Library, Cape Tozon.

Christchurch, New Zealand : The Library of Canterbury College, Cbristchurch.

Colomвo : University College, Colombo, Ceylon.

Dunedin, New Zealand : The Library of Otago University, Dunedin.

Grahamstown, S. Africa: The Library of Rhodes University College, Grabamstown.

Hobart, Tasmania: The University of Tasmania.

Johannesburg, S. Arrica: The Public Library, Johanneslurg.

Kingston, Ontario: The Library of Queen's University, Kingston.

London, Ontario: The Western University Library, London.

Melbourne, Australia: The Public Library, Melbourne.

Melbourne, Australia: The Library of the University, Melbourne.

Montreal, Canada: The Library of the McGill University, Montreal.

Point Grey, Britisil Columbia: The University Library, Point Grey. 
xxviii

Pretoria, Transvaal, S. Africa: The T.U.C. Co-operative Supply Association L.td., Transvaal University College, Pretoria.

Strllen bosch, S. Africa : The University of Stellenbosch, Stellenbosch.

Sydney, New South Wales: The Public Library, Sydney.

Sydney, New South Wales : The University Library, Sydney.

Toronto, Canada : The University Library, Toronto.

\section{THE UNITED STATES OF AMERICA.}

Amherst, Mass : The Library of Amherst College, Amberst.

Andover, Mass: The Library of Phillips Academy, Brechin Hall, Andovcr.

An n Arbor, Michigan : The Library of Michigan University, Ann Arbor.

Athens : Carnegie Library, Ohio University, Athens.

Aukora, New York : The Library of Wells College, Aurora.

Austin, Texas: The Library of Texas University, Austin.

Baltimore, Maryland : The Library of Johns Hopkins University, Baltimste.

Baltimore, Maryland: The William Welch Medical Library, Baltimore.

Berkeley, California : The Library of the University of California, Berkeley.

Bethlehem: Lehigh University Library, Betblebem, $P a$.

Bloomington, Ind. : Indiana University Library, Bloomington.

Boston, Mass: The Library of the Athenaeum, Boston.

Bosron, Mass : The Library of the Museum of Fine Arts, Boston.

Boston, Mass.: The Public Library, Boston.

Boulder, Colo. : The Library of Colorado University, Boulder.

Brookryn, New York : The Museum, Brooklyn Institute of Arts and Sciences, Brooklyn.

Brunswick, Maine: The Library of Bowdoin College, Brunswick.

Bryn Mawr, Pa.: The Library of Bryn Mawr College, Bryn Mawr.

Burlington: The University of Vermont, Burlington.

Cambridge, Mass.: The Library of Harvard University, Cambridge.

Carlisle, Penn.: The Library of Dickinson College, Carlisle.

Chapel Hill, N. Carolina : The Library of the University of N. Carolina, Chapel Hill.

Charlotresville, $V_{A}$ : The Library of the University of Virginia, Charlottesville.

Chicago, Ill. : Loyola University Library, Cbicago.

Chicago, Ill.: The Newberry Library, Cbicago.

Chicago, Ill : The Library of the University, Chicago.

Cincinnati, Ohio: The Library of the University, Cincinnati.

Cleveland, Onio: The Library of Adelbert College, Cleveland

Cleveland, Ohio: The Public Library, Cleseland.

Clinton, New York: The Library of Hamilton College, Clinton.

Columbia, Mo.: The Library of Missouri University, Columbia.

Columbus, Онiо : The State University, Obio.

Durнam, N.C.: The Library of Duke University, Durbam, N.C.

Easton, $\mathrm{PA}_{\mathrm{A}}$ : The Library of Lafayette College, Easton.

Evanston, Ill : The Library of the North Western University, Evanston.

Grinnell, Jowa: The Library of Grinell College, Iowa.

Hanover: Dartmouth College Library, Hanover, New Hants.

Haverford : Haverford College Library, Haverford, Pennsylvania.

In DIANAPOLIS, IND.: Butler College Library, Indianapolis.

Iowa City : The Library of the State University, Iowa.

IthacA, New York: The Library of Cornell University, Itbaca.

Lawrence, Kansas: The Library of Kansas University, Lawrence.

Lincoln, Nebraska: The Library of Nebraska University, Lincoln.

Los Angeles, California : The Library of California University, Los Angeles.

Madison, Wiscovsin : The Library of the University of Wisconsin, Madison.

Middeetown, Conn.: The Library of the Wesleyan University, Middletown.

Minneapolis, Minn.: The Library of the University of Minnesota, Minneapolis. 
New Haven, Conn.: The Library of Yale Universitv, New Haven.

New York: The American Geographical Society, 156th Street, Broadway, New York.

New York : The Library of Columbia University, New $r_{\text {ork. }}$

New York: The Metropolitan Museum of Art, New York.

New York : The Public Library, New York.

New York: The University Library, University Heights, New York.

Norman, Oklahoma: The Library of Oklahoma University, Norman.

Northampton, Mass : The Library of Smith College, Northampton.

Oberlin: Oberlin College, Oberlin.

Ohio: Cleveland Public Library, Obio.

Philadelphia, Pa.: The Free Library, Pbiladelpbia.

Philadelphia, Pa.: The Library Company, Pbiladelphia.

Philadelphia, PA. : The Library of the University of Pennsvlvania, Pbiladelpbia.

Philadelphia, PA.: The Library of the University Museum, Pbiladelpbia.

Pittsburg, Pa.: The Carnegie Library, Pittsburg.

Pough eefsie, New York: The Library of Vassar College, Poughkeepsie.

Princeton, New Jersey: The University Library, Princeton.

Providence, Rhone Island: The Library of Brown University, Providence.

Rochester, N.Y.: The Library of Rochester University, Rochester.

St. Louis, Missouri : The Library of Washington University, St. Louis.

Schenectady, New York: Union College Library, Schenectady.

Seattle: University of Washington Library, Seattie, Washington.

South Hadley, Mass: The Library of Mount Holyoke College, South Hadley.

Stanford, California : The Library of the University, Stanford.

Swarthmore, Pa: The Library of Swarthmore College, Swartbmore.

Terre Haute, Ind : The Library of Indiana State Normal School, Torre Haute.

URBANA, ILL : The Library of the University of Illinois, Urbana.

Washington: The Library of the Catholic University of America, Wasbington, D.C.

Washington : The Library of Congress, $W$ asbington.

Wellesley, Mass : The Library of Wellesley College, $W_{\text {ellesley. }}$

\section{AUSTRIA.}

Graz: Archäologish-epigraphisches Seminar der Universität, Graz.

VIENNA : Archäologisch-epigraphisches Seminar der Universität, Vienna.

\section{BELGIUM.}

Brussels : Bibliothèque royale de Belgique, Rue du Musée, Brussels.

Brussels: Musées Royaux du Cinquantenaire, Brussels.

Brussels : La Société des Bollandistes, Brussels.

Ghext : L'Université de Gand, 2 Fossé d'Othon, Ghent.

Lí́ge : The Faculty of Philosophy and Letters at the University, Liege.

\section{BULGARIA.}

Sofra : I'Institut archéologique Bulgare, Rakowska i27, Sofia.

$$
\text { CHINA. }
$$

Peking : Metropolitan Library, Peking.

\section{CZECHOSLOVAKIA.}

Prague: The Public University Library, Prague.

\section{DENMARK.}

Copenhagen : Kongelike Bibliothek, Copenbagen.

Copenhagen: Ny Carlsberg Glyptotek, Copenhagen. 
EGYPT.

Alexandria : La Musée Greco-Romain, Alexandria.

Carro: La Bibliothèque de l'Université égyptienne, Abassieh, Carro.

\section{ESTONIA.}

Tartu (Dorpat) : Institute of Classical Archaeology, The University, Tartu.

\section{FRANCE.}

Algiers, N. Africa : La Bibliothèque de l'Université, Alger.

Grenoble : La Bibliothèque de l'Université, Grenoble, Isère.

ParIs : La Bibliothèque de l'Université à la Sorbonne, Paris.

Paris: La Bibliothèque de l'Ecole Normale Supérieure, Parts.

Paris : La Bibliothèque Nationale, Paris.

Paris : La Bibliothèque de l'Institut de France, Paris.

Paris : La Bibliothèque d'Art et d'Archéologie, Paris.

Strasbourg : Bibliothèque nationale et universitaire, Strasbourg.

\section{GERMANY.}

Berlin : Arch. Seminar d. Universität, Berlin.

BerLin : Institut für Altertumskunde, Berlin.

BERLIN : Universitätsbibliothek, Beriin.

BerLin : Preussische Staatsbibliothek, Bcrlin.

BresLaU : Universitätsbibliothek, Breslau.

FReIbURG-I.-BReIsGAU : Universitätsbibliothek, Freiburg-i-Breisgau.

Girssen : Universitätsbibliothek, Giessen.

Göttingen : Universitätsbibliothek, Göttingen.

GreIFswaLd : Universitätsbibliothek, Greifswald.

Hamburg : Stadt-u. Universitätsbibliothek, Hamburg.

Heidelarerg: Universitätsbibliothek, Heidelberg.

KIEL: Universitätsbibliothek, Kiel.

Koeln-Bayenthal: Arch. Institut d. Universität, Koeln-Bayentbal.

Koeln-Rhein : Universitat-u.-Stadtbibliothek, Koeln-Rhein.

KöNIGSBERG : Universitätsbibliothek, Königsberg.

LeIPZIG : Arch. Institut d. Universität, Leipzig.

LeIPZIG : Universitätsbibliothek, Leipzig.

Marburg : Universitätsbibliothek, Marburg.

Muvicu: Bayr. Staatsbibliothek, Munich.

Munich: Universitätsbibliothek, Munich.

MÜNSTER : Universitätsbibliothek, Münster.

Rosтock : Universitätsbibliothek, Rostock.

T IFR: Provinzialmuseum, Trier.

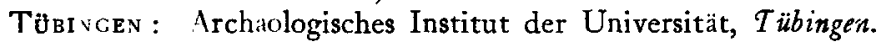

T'UвINGEN : Universititsbibliorhek, $\tau$ übingen.

Würzßuks: Kunstgeschichtliches Museum der K. Julius-Maximilians Universität, IF'̈̈̈r $\approx$ burg

WürZBURG : Universitätsbibliothek, Würzburg.

\section{GREECE.}

Atuevs: American School of Classical Studies, Athens.

Aturve: Deutsches archaeologisches Institut, Phidias-str. 1, Athens.

Atrens: L'école française d'Athènes, Athens. 
HOLLAND.

The Hague : Koninklijke Bibliotheek, The Hague.

LEEUWARDEN : Buma Bibliotheek, Leeuwarden.

LEIDEN : Bibliotheek der Rijks-Universiteit, Leiden.

Nymegen : Rijks-Museum G. M. Kam, Nymegen.

UTREснт: Bibliotheek der Universiteit, Utrecht.

\section{HUNGARY.}

Budapest : The Museum in Aquincum, Budapest.

BudAPFis : University Library, Budapest.

\section{ITALY.}

Cagliari (Sardegna): Biblioteca della Facultà di Lettere e Filosofia, R. Università, Cagliari.

Città del Vaticano: Biblioteca Apostolica Vaticana, Citta del Vaticano.

Florence: R. Biblioteca Nazionale Centrale, Florence.

Milan : Civici Istituti d'Arte, Castello Sporzesco, Milano.

Padua : Gabinetto d'Archeologia della R. Università, Padua.

PAviA : Biblioteca della Facultà di Filosofia e Lettere, R. Università, Pavia.

Rome: The American Academy in Rome, Porta S Pancrazio, Rome

Rome : Deutsches Archäologisches Institut, Via Sardegna, 79, Rome (25).

Roxe: Gabinetto Epigrafia, R. Università, Rome.

Rome: La Biblioteca Nazionale Vittorio Emanuele, Rome.

Rome: Ufficio Scavi di Ostia, Rome.

Turin : La Biblioteca Nazionale. Turin.

JAPAN.

Tокуо: The Imperial University Library, Tokyo.

OsLo: Universitetsbibliotek, Oslo.

\section{NORWAY.}

PALESTINE.

Jerusalem: The British School of Archaeology, ferusalem.

POLAND.

Cracow : Archaeological Institute, I2 St. Anne Street, Cracow.

RUSSIA.

Leningrad : Gosudarstvennoi Akademii Istorii Materialnoi Kultury, Ul. Chalturini 5, Leningrad.

Leningrad: The Hermitage Museum Library, Chalturinastr. 35, Leningrad.

\section{SWEDEN}

Gothendurg : Stadbiblioteket, Göteborg.

Lund: The Library of the University, Lund,

Sтоскноцм : The Royal Library, Stockbolm.

Uppsala: The Library of the University, Uppsala.

\section{SWITZERLAND.}

Lausanne: M. Charles Favez, L'Association de Lectures philologiques, 39 Bvà. de Grancy, Lausanne.

ZürICH : Zentralbibliothek, Zürich 
$\mathrm{xxxi1}$

\section{EXCHANGES OF PUBLICATIONS.}

The following is a list of Societies, Institutions, etc, with which exchanges of publications have been arranged:

\section{FRANCE.}

Paris : L'Association Guillaume Budé, 95, Boulevard Raspail, Parts.

\section{GREAT BRITAIN.}

Carliste: Cumberland and Westmorland Antiquarian and Archaeological Society, Tullie House, Carlisle.

Devizes: The Wiltshire Archaeological and Natural History Society, The Museum, Devizes.

Edinburgh : The Society of Antiquaries of Scotland, Queen Street, Edinburgh.

Hotywet. : The Cambrian Archaeological Association, Whitford, Holywell.

Leens : The Yorkshire Archaeological Society, ro Park Place, Leeds.

Lewes: The Sussex Archaeological Society, Lewes.

London: The Society of Antiquaries, Burlington House, Piccadilly, London.

Newcastle-upon-Tyne: The Society of Antiquaries, Nevocastle-upon-Tyne.

\section{GERMANY.}

BerLiN : Zeitschritt für Ethnologie.

BrrLin : Bayerisch. Numismat. Gesellschaft, Karlstrasse io, Munich.

Bonn: Provinzialmuseum, Bonn.

Bon : Verein von Altertumsfreunden im Rheinlande, Bonn.

Frankfurt a. M : Deutsches Archäologisches Institut, strasse I2, Palmgarten, Frankfurt am $M$.

Marnz: Röm.-Germanisch. Centralmuseum, Mainz.

Munich : Archäologisches-Seminar der Universität, Munich.

\section{HUNGARY.}

Budapest: Magyar Nemzeti Museum.

ITALY.

Milan : Historia, Via Moscova, I7, Milan.

Rome: Accademia di Romania, Via Emilio di Cavaliere, Rome (36).

Rome: The Library of the British School, Valle Giulia, Rome.

Rome: L'École française de Rome.

Rome: Pontificio Istituto Biblico, Piazza della Pilotta 35, Rome.

Roma : Rivista di Archeologia Cristiana, Via Napoleone III ${ }^{1}$, Rome.

Roma : Rivista di Archeologia e Storia dell'Arte. Palazzo Venezia, 3, Rome.

Turin : Rivista di Filologia e d'Istruzione Classica (Casa Editrice Giovanni Chiantore.

Via Vittorio Amedeo ii, I8, Turin. ${ }^{3}$ )

Bucharest: The Academia Română.

$$
\text { ROUMANIA. }
$$

SPAIN.

Barcelona: Institut d'Estudis Catalans, Palau de la Diputació, Barceiona. 